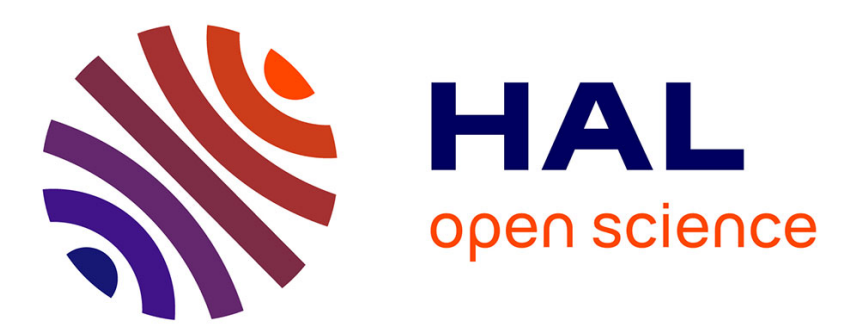

\title{
Asymmetric iodine catalysis-mediated enantioselective oxidative transformations
}

Aurélie Claraz, Géraldine Masson

\section{To cite this version:}

Aurélie Claraz, Géraldine Masson. Asymmetric iodine catalysis-mediated enantioselective oxidative transformations. Organic \& Biomolecular Chemistry, 2018, 16 (30), pp.5386-5402. 10.1039/C8OB01378K . hal-02410604

\section{HAL Id: hal-02410604 https://hal.science/hal-02410604}

Submitted on 13 Dec 2020

HAL is a multi-disciplinary open access archive for the deposit and dissemination of scientific research documents, whether they are published or not. The documents may come from teaching and research institutions in France or abroad, or from public or private research centers.
L'archive ouverte pluridisciplinaire HAL, est destinée au dépôt et à la diffusion de documents scientifiques de niveau recherche, publiés ou non, émanant des établissements d'enseignement et de recherche français ou étrangers, des laboratoires publics ou privés. 


\section{Journal Name}

\section{ARTICLE}

\section{Asymmetric iodine catalysis-mediated enantioselective oxidative transformations}

Received 00th January 20xx Accepted 00th January 20xx

DOI: $10.1039 / \times 0 \times x 00000 x$

www.rsc.org/

\begin{abstract}
Aurélie Claraz* and Géraldine Masson*
The implementation of chiral iodine catalysis has tremendously been developed in the field of asymmetric synthesis during the last decade. It enables the stereoselective creation of $\mathrm{C}-\mathrm{O}$ as well as $\mathrm{C}-\mathrm{C}, \mathrm{C}-\mathrm{N}$ and $\mathrm{C}-\mathrm{X}(\mathrm{X}=$ halogen) bonds through oxidative transformations. Thanks to the low toxicity and ease of handling of iodine compounds, this strategy offers many advantages over classical metal-catalyzed oxidations with chiral ligands. The approaches rely on iodine $(\mathrm{I} / \mathrm{III})$ or $(-1 /+\mathrm{I})$ catalysis by using a chiral aryliodine or ammonium iodide respectively in combination with a suitable terminal oxidant. As such, the design of iodine compounds with central, axial or even planar chirality has allowed to achieve high enantioselectivities. The goal of this review is to cover the different chiral iodine compounds-catalyzed oxidative transformations including $\alpha$-functionalization of carbonyl compounds, dearomatization of phenol derivatives and difunctionalization of alkenes which should demonstrate that iodine catalysis has now found its place in the realm of asymmetric organocatalysis.
\end{abstract}

\section{Introduction}

lodine catalysis has emerged as a versatile and eco-friendly strategy to perform oxidative transformations allowing for instance couplings with formation of a $\mathrm{C}-\mathrm{O}, \mathrm{C}-\mathrm{N}, \mathrm{C}-\mathrm{C}$ or $\mathrm{C}-\mathrm{X}$ ( $\mathrm{X}=$ halogen) bond. ${ }^{1}$ In order to describe mechanisms of such reactions, synthetic chemists frequently borrow the terms of oxidative additions, ligand exchanges and reductive eliminations to the field of metal-mediated processes. Nevertheless, iodine compounds are mild, stable, easy to handle and eco-compatible reagents, advantageously competing with heavy metals. As such, it is not surprisingly that iodine catalysis has attracted the interest of the synthetic community in the field of asymmetric synthesis with the aim to develop a greener and more environmentally benign chemistry. ${ }^{2}$ Up to now, asymmetric iodine catalysis-mediated oxidative transformations rely on the catalytic use of either hypervalent organoiodine (III) reagents (also called $\lambda^{3}$-iodanes) or organic hypoiodites (I) as chiral oxidants. In the first case, chiral organoiodanes (III) ${ }^{3}$ are generated in situ from aryliodine precatalysts and a stoichiometric amount of achiral co-oxidant (Figure 1). The first enantioselective catalytic transformation was reported in 2007 by Wirth et al. for the $\alpha$-oxytosylation of ketones by using an aryliodine species bearing a chiral moiety ortho to the iodine atom in the aromatic ring. ${ }^{4}$ Since this original report, numerous chiral aryliodoarene-mediated enantioselective oxidative transformations through iodine (I/III) catalysis have been investigated. ${ }^{5}$ Besides cost reductions and atom economy, the development of catalytic processes allows to avoid the preliminary preparation and isolation of the chiral hypervalent orgaonoiodine (III) reagent, since this reactive species is generated in situ from an aryliodine. Obviously, this feature can be advantageous during the design of new chiral precatalysts, especially for unstable hypervalent organoiodine (III) derivatives. The currently known enantiopure precatalysts of hypervalent organoiodine (III) compounds exhibit either $\mathrm{C}_{1}$ or $\mathrm{C}_{2}$-symmetry with central, axial or even planar chirality (Figure 1). 


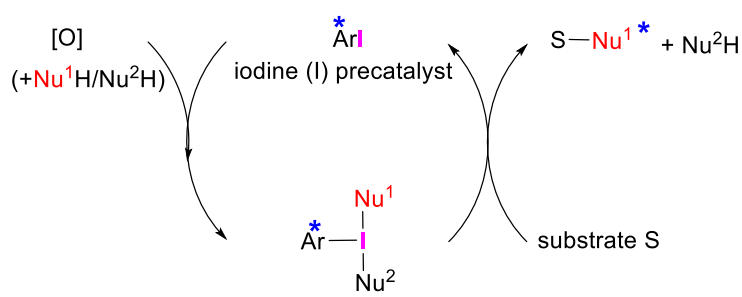

hypervalent iodine (III)

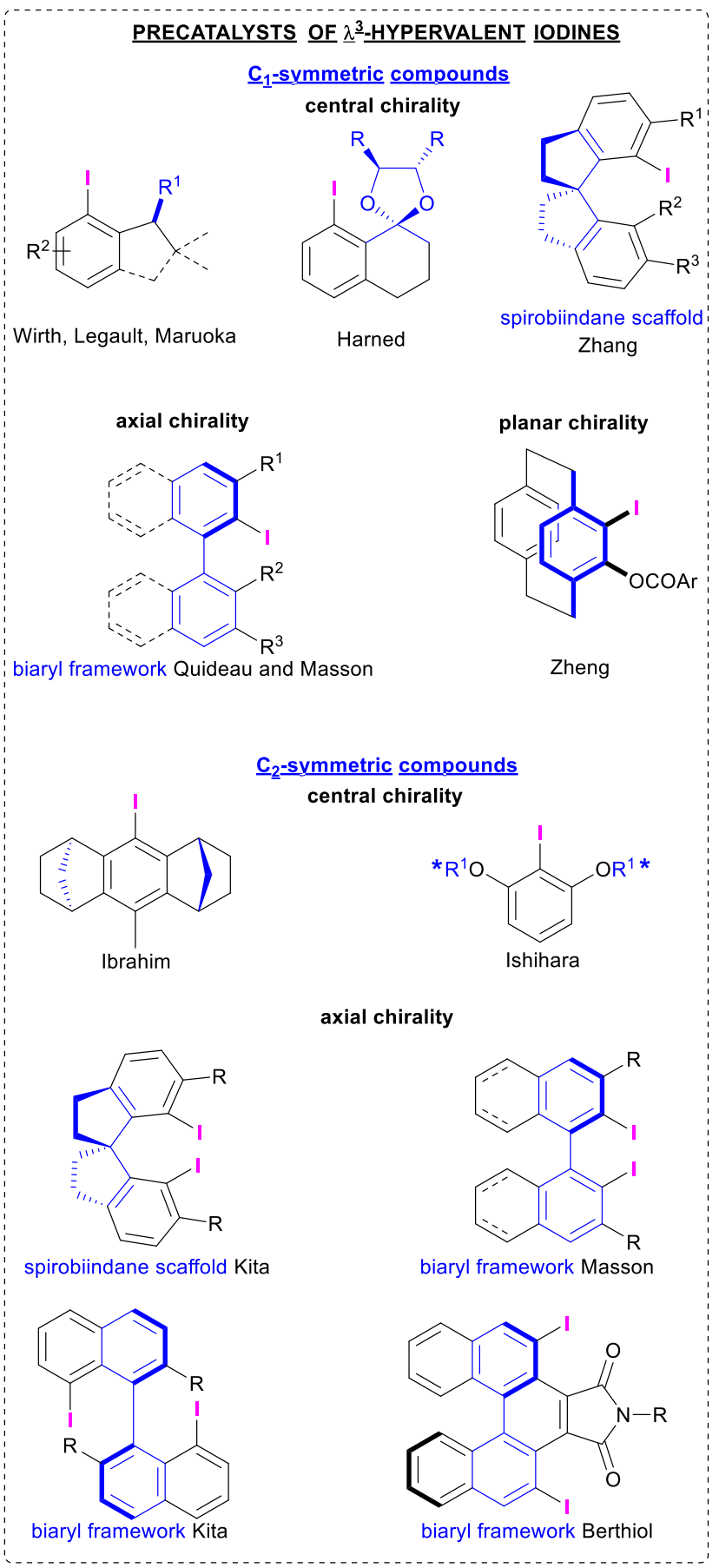

Figure 1 Concept of iodine (I/III)-enabled enantioselective oxidative transformations and general structure of chiral ayliodine precatalysts.

On the other hand, chiral hypoidites (I) are generated in situ from chiral organic iodides (-I) (typically chiral ammonium iodides that are traditionally used in asymmetric phase transfer catalysis), ${ }^{6}$ and a stoichiometric amount of achiral co-oxidant (Figure 2$)^{7}$
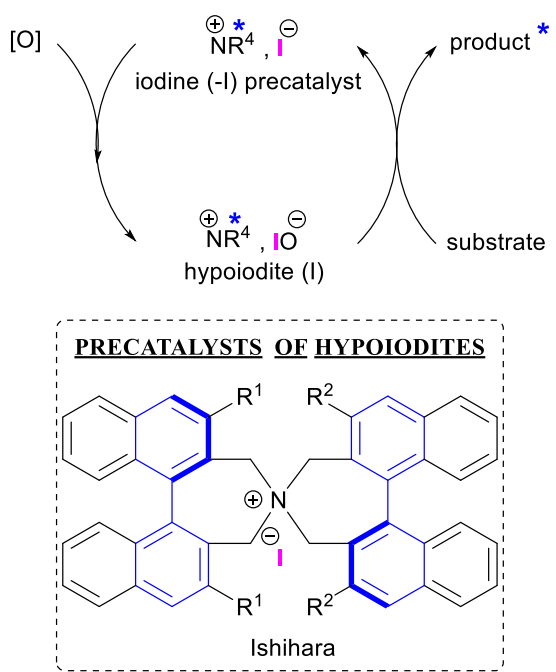

Figure 2 Concept of iodine $(-1 / I)$-enabled enantioselective oxidative transformations and general structure of chiral ammonium iodide precatalysts.

In both catalytic cycles depicted above, (re)oxidation of the iodine atom of a precatalyst in low valent state to higher oxidation state is the key step. ${ }^{8}$ The choice of this stoichiometric achiral oxidant is critical for the success of the reaction. In an ideal fashion, it should not interact with the starting material or, at least, reoxidation of the precatalyst should be faster than the rates of undesired side reactions. In asymmetric processes, peroxides are the most commonly used oxidants that satisfy with more or less success these requirements.

To get cleaner reactions along with highest levels of enantiocontrol, a careful optimization of other classical reaction parameters such as solvent, temperature and the use of additives must be undertaken.

The focus of this review is to summarize the use of chiral iodine compounds in catalytic enantioselective oxidative processes with the aim of highlighting both catalysts design and synthetic applications. As such, we hope that this survey will help the reader in designing new chiral iodine precatalysts and applying those to new catalytic processes. For the sake of clarity, this review has been divided into different sections according to the kind of oxidative transformation considered:
a. $\alpha$-functionalization of carbonyl compounds,
b. dearomatization of phenol derivatives,
c. functionalization of alkenes. 


\section{2. $\alpha$-Functionalization of carbonyl compounds}

Hypervalent iodine compounds enable the introduction of a nucleophile at the $\alpha$-position of a carbonyl compound. As such, numerous efforts have been made towards the development of enantioselective versions to take advantage of this inversion of polarity (umpolung reaction).

\section{1. $\alpha$-Oxytosylation}

The enantioselective $\alpha$-oxytosylation of ketones mediated by chiral hypervalent iodines gives access to synthetically useful enantioenriched carbonyl compounds. As mentioned above, the first catalytic version of this reaction was reported in 2007 by Wirth et al. Inspired by the works of Togo et al. who demonstrated a catalytic racemic version of this reaction, ${ }^{9}$ Wirth et al. employed organoiodane I with central chirality as the chiral inducer, meta-chloroperoxybenzoic acid (mCPBA) as the stoichiometric oxidant and $p$-toluenesulfonic acid monohydrate $\left(\mathrm{TsOH} \cdot \mathrm{H}_{2} \mathrm{O}\right)$ as a source of tosylate nucleophiles (Scheme 1). ${ }^{4}$ Contrarily to the process under stoichiometric conditions, ${ }^{10}$ the catalytic transformation needs to be carried out at room temperature, which suggests that oxidation of the iodoarene is the rate determining step in the catalytic cycle. Noteworthily, no racemization of the product in the reaction medium was observed. Nevertheless, only moderate enantioselectivities were obtained in this difficult transformation. From a mechanistic point of view, the authors proposed the in situ generation of a chiral Koser's-type reagent from I, mCPBA and $\mathrm{TsOH} \cdot \mathrm{H}_{2} \mathrm{O}$, furnishing either intermediate $\mathbf{3}$ or 4. Subsequent nucleophilic addition of a tosylate anion on $\mathbf{3}$ (path A) or 4 (path B) via $\mathrm{S}_{\mathrm{N}} 2^{\prime}$ and $\mathrm{S}_{\mathrm{N}} 2$ type mechanisms would both provide enantioenriched ketone $\mathbf{2}$ along with the regeneration of aryliodine $\mathbf{I}$. Interestingly, the reaction is generally clean and the $\alpha$-oxybenzoyl ketones which would result from the nucleophilic addition of the mCBA by-product were not formed.

Importantly, the authors pointed out the need of a chelating group at the benzylic position on the ortho substituent of the iodine atom in $\mathbf{I}$ to get the highest possible levels of enantioselectivity. Based on this observation, they synthesized compound II, having a chiral ester moiety as the chelating function at the benzylic position instead of the methoxy group and resulting in an increase of enantioselectivity up to $39 \%$ during the $\alpha$-oxytosylation of propiophenone (Scheme 2). ${ }^{11}$ In 2010, the same group prepared derivative III, bearing a chiral ester at the ortho position of the iodine, and leading to lower enantioselectivity. ${ }^{12}$

In 2012, the group of Legault et al. developed chiral aryliodine IVa bearing a chiral oxazoline as a coordinating motif and a methyl at the ortho and ortho'-positions of the iodine. ${ }^{13}$ Premixing catalyst, substrate, and $\mathrm{TsOH}$, followed by slow addition of mCPBA slightly enhanced both yield and selectivity during the $\alpha$-oxytosylation of propiophenone. According to computational analyses, this procedure would favor the formation of the key reactive iodane 5 by minimizing $N$ oxidation of the oxazoline moiety through $\mathrm{N}$-protonation. ${ }^{14}$ Noteworthily, corresponding aryliodine IVb without the ortho'- methyl substituent is not able to promote the reaction as protonation of $\mathbf{I V b}$ is much more difficult. The best selectivity was finally obtained after the introduction of a chlorine atom para to the iodine (IVc).

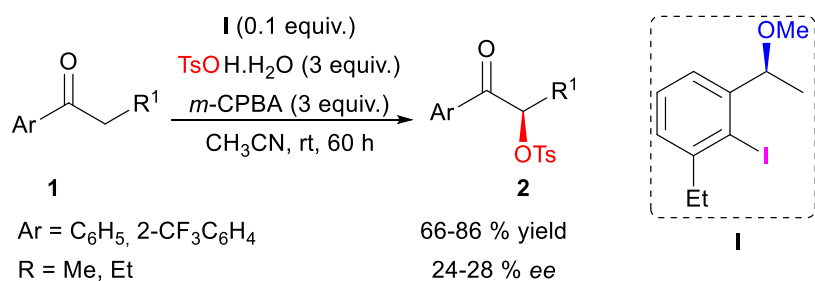

$\mathrm{R}=\mathrm{Me}, \mathrm{Et} \quad 24-28 \%$ ee
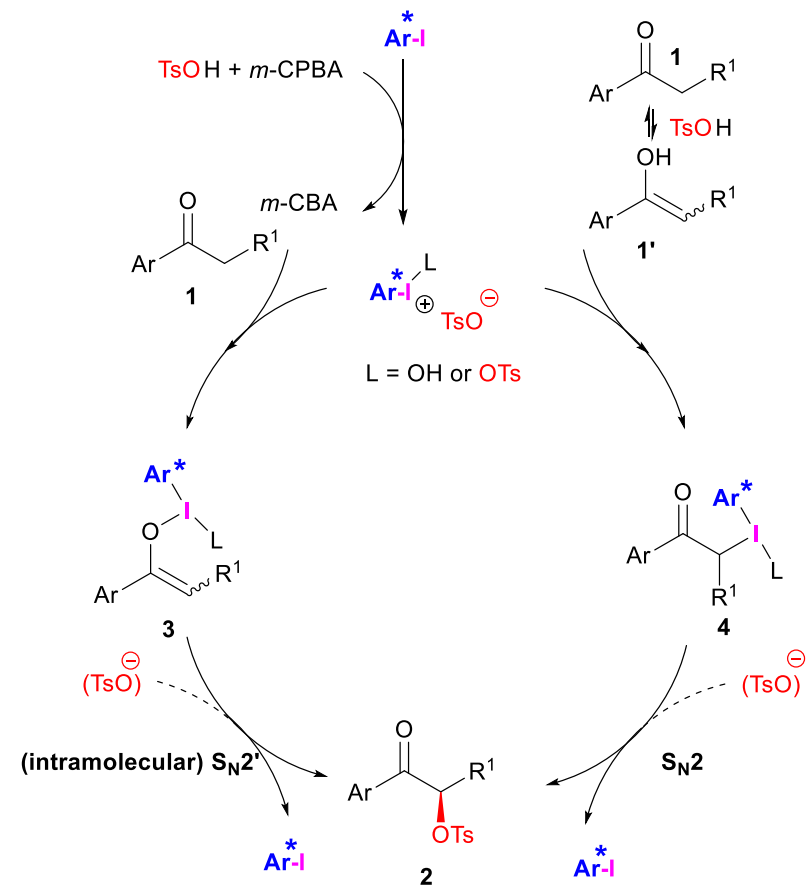

Scheme 1 First catalytic enantioselective $\alpha$-oxytosylation of ketones. 


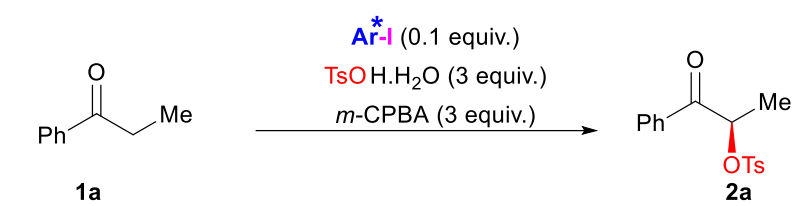

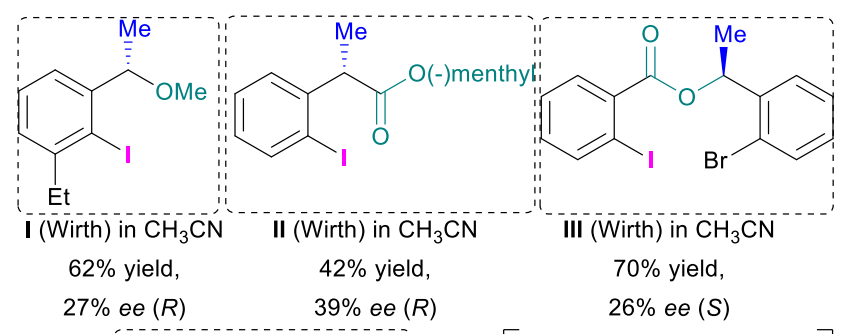

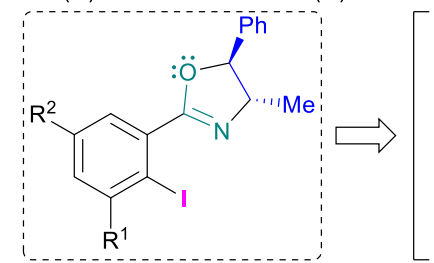

IV (Legault) in $\mathrm{CH}_{3} \mathrm{CN} / \mathrm{CH}_{2} \mathrm{Cl}_{2} 1: 1$

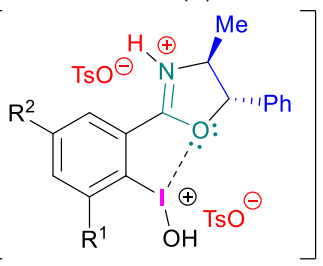

5

IVa $\mathrm{R}^{1}=\mathrm{Me}, \mathrm{R}^{2}=\mathrm{H} ; 72 \%$ yield, $44 \%$ ee $(R)$

IVb $\mathrm{R}^{1}=\mathrm{H}, \mathrm{R}^{2}=\mathrm{H} ; 3 \%$ yield, ee nd

IVc $\mathrm{R}^{1}=\mathrm{Me}, \mathrm{R}^{2}=\mathrm{Cl} ; 80 \%$ yield, $44 \%$ ee $(R)$

Scheme 2 New ortho-substituted iodobenzene precatalysts in the $\alpha$ oxytosylation.

The enantiopure spirobiindane scaffold has also been exploited as a source of chirality in the $\alpha$-oxytosylation of carbonyl compounds. In 2011, Zhang et al. have synthesized chiral iodoarene $\mathbf{V a}$ in 7 steps ( $28 \%$ yields) from expensive commercially available $(S)-1,10$-spirobiindane-7,7'-diol. ${ }^{15}$ In the presence of $\mathrm{mCPBA}$ as stoichiometric oxidant in acetonitrile, the $\alpha$-oxytosylation of propiophenone $\mathbf{1}$ afforded product $\mathbf{2}$ in $65 \%$ yield and $30 \%$ ee. Enantioselectivity has been improved to $53 \%$ ee by simply switching solvent from acetonitrile to ethyl acetate (Scheme 3). In contrast to Wirth's report, slight racemization of the product was observed in the reaction medium. It is worth mentioning that lower enantioselectivity was obtained with $\mathrm{C}_{2^{-}}$ symmetrical chiral diiodoarene $\mathbf{V b}$. Introduction of other chelating and/or sterically demanding substituents on the spirobiindane scaffold close to the iodine atom (4-, 6-, and 7'positions) did not improve enantioselectivity. This reaction scope has been extended to other aromatic ketones and sulfonic acids, yielding corresponding $\alpha$-functionalized ketones in up to $56 \%$ ee.

During their quest for a suitable precatalyst in the catalytic enantioselective $\alpha$-oxytosylation of propiophenone, Wirth et al. have tested 2,2'-diiodo-1,1'-binaphthyl (BINI) VI.Erreur ! Signet non défini. Poor enantioselectivity was obtained indicating that further tuning of this axially chiral backbone was required. In 2013, Berthiol et al. introduced new $\mathrm{C}_{2}$-symmetric 3,3'-diiodoBINOL-fused maleimide derivatives $\mathbf{V I I a}^{16}$ and $\mathbf{V I I b}^{17}$ that showed a stereochemical efficiency comparable to the best results previously reported (Scheme 4). In 2017, Masson et al. reported an axially chiral, non $\mathrm{C}_{2}$-symmetric iodoarene VIII bearing a sulfone substituent as a potentially chelating group of the iodine atom. This catalyst afforded $(R)-\mathbf{2 a}$ in $75 \%$ yield and
$67 \%$ ee, which is the best enantioselectivity obtained up to date from 1a under catalytic conditions. ${ }^{18}$ Noteworthily, this catalyst is easily prepared in few steps involving a Palladium-catalyzed stereoselective cross-coupling. ${ }^{19}$

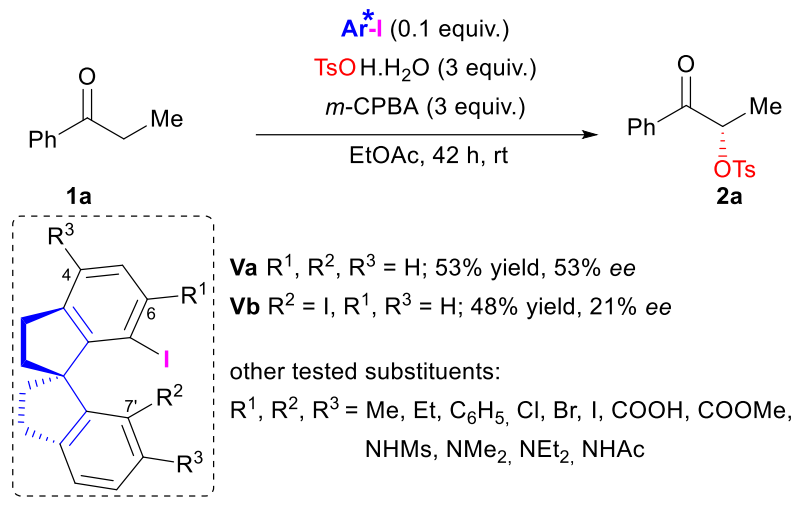

Scheme 3 Spirobiindane scaffold in the $\alpha$-oxytosylation of ketones.

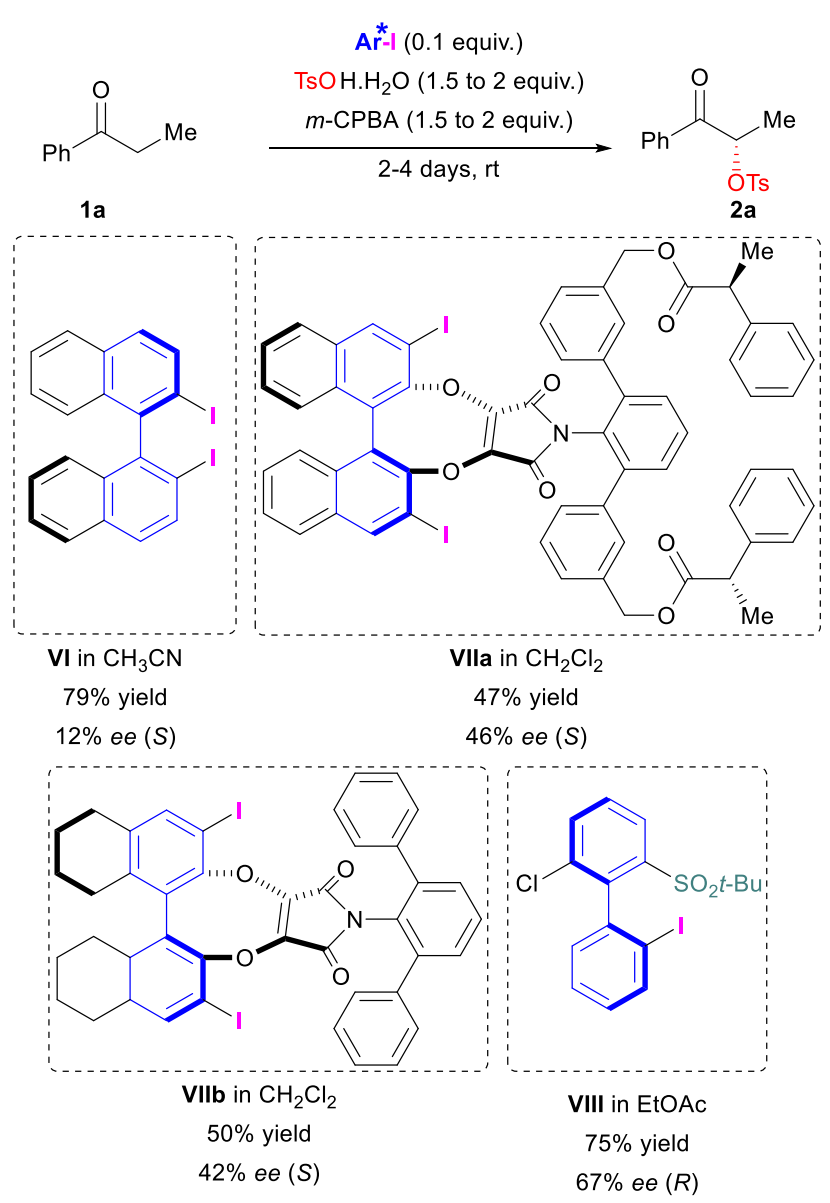

Scheme 4 Axially chiral biaryl precatalysts in the $\alpha$-oxytosylation of ketones.

Despite the great advances which have been made, the direct $\alpha$-oxytosylation of ketones still suffers from moderate enantioselectivities. The competition between the two different routes depicted in scheme 1 might be responsible of this limited enantiocontrol. Especially, the great distance between the iodoarene group in $\mathrm{O}$-iodonium enolate $\mathbf{3}$ and the forming stereocenter can make efficient stereoinduction very 
difficult. ${ }^{20}$ The lack of control in the geometry of enol $\mathbf{3}$ might be also problematic. To avoid the passage through the $O$ bonded intermediate $\mathbf{3}$ and favor the $\boldsymbol{C}$-bonded intermediate $\mathbf{4}$, Legault et al. considered $\alpha$-oxytosylation of enolacetate 6 (Scheme 5). With such a strategy, $C_{2}$-symmetric 2idodoresorcinol derivative IX offered the $\alpha$-oxytosylated ketone 2 in good yield and with high enantioselectivity. ${ }^{21}$ It is worth pointing out that very poor enantioselectivity was obtained during the direct $\alpha$-oxytosylation of ketone $\mathbf{2}$ with the same kind of catalyst. This observation supports two different mechanisms between the $\alpha$-oxytosylation of ketone and enol acetate: $S_{N} 2^{\prime}-$ type reductive elimination of a $O$-enolate intermediate in the former case providing low enantiocontrol in the former case and highly enantioselective $\mathrm{S}_{\mathrm{N}} 2$ substitution of a $C$-enolate in the latter case. ${ }^{20}$

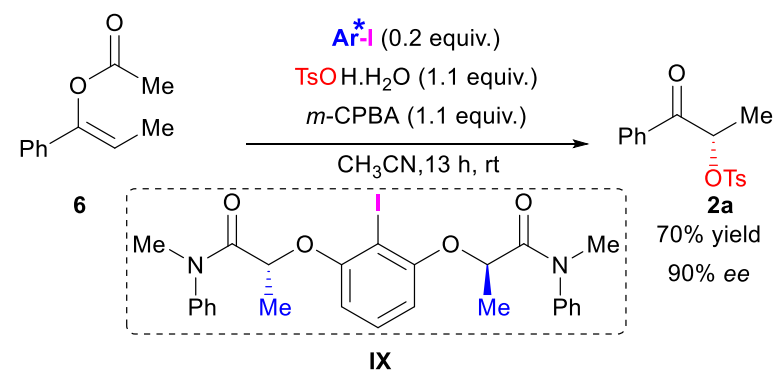

Scheme $5 \alpha$-oxytosylation of enol acetates.

In 2016, Xiong, Coeffard et al. investigated the $\alpha$ oxytosylation of $\alpha$-substituted $\beta$-ketoesters 7 by using $C_{2}-$ symmetric iodoarene IX as chiral precatalyst in combination with mCPBA as stoichiometric oxidant at $-20^{\circ} \mathrm{C}$ in chloroform (Scheme 6). ${ }^{22}$ Corresponding products 8 with a quaternary stereogenic center were obtained in poor yields albeit respectable enantiomeric excesses (up to $37 \%$ yield and $62 \%$ ee). Interestingly, 3 equivalents of trifluoroacetic acid (TFA) as additive were able to increase enantioselectivity. ${ }^{23}$

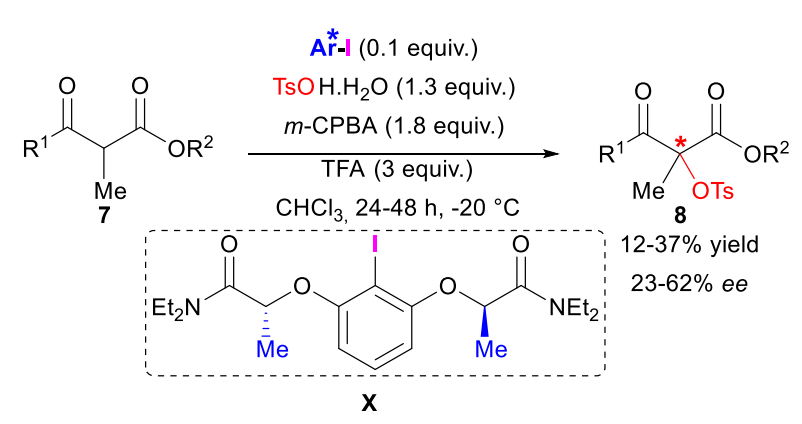

Scheme $6 \alpha$-oxytosylation of $\alpha$-substituted $\beta$-ketoesters.

\subsection{Intramolecular $\alpha$-oxygenation}

The hypervalent iodine catalyzed $\alpha$-oxygenation of carbonyl compounds has been extended by Moran et al. to an intramolecular version via lactonization of 5-oxo-5phenylpentanoic acid 9 (Scheme 7). ${ }^{24}$ Inspired by the works of Legault et al., ${ }^{13}$ the authors synthesized ortho-tolyliodine $\mathbf{X I}$ bearing a pseudoephedrine-based chiral amide as chelating
Lewis agent. In the presence of substoichiometric amount of mCPBA and $p \mathrm{TsOH}, 5$-benzoyldihydrofuran-2(3H)-one $\mathbf{1 0}$ was obtained with moderate yield and enantioselectivity.

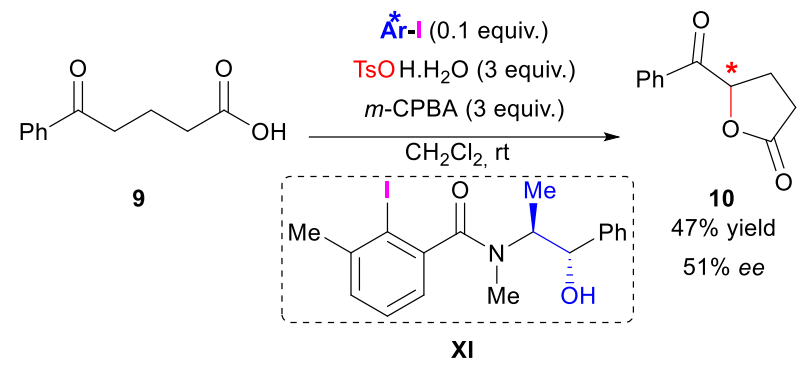

Scheme 7 Oxidative cyclization of 5-oxo-5-phenylpentanoic acid.

In 2010, an elegant catalytic enantioselective oxidative cyclization of ketophenols $\mathbf{1 1}$ to the five-membered ring products 2-acyl-2,3-dihydrobenzofurans $\mathbf{1 2}$ has been developed by Ishihara et al. (Scheme 8a). Excellent yields and enantioselectivities at very low catalyst loading were obtained Their approach relied on the use of a in situ generated chiral hypoiodite $\left[\mathrm{IO}^{-}\right.$catalyst from the corresponding quaternary ammonium iodide precatalyst XII with hydrogen peroxide or tert-butyl hydroperoxide (TBHP) as environmentally benign cooxidants. ${ }^{25}$ The unstable catalytic active species hypoiodite has been detected by a series of Raman spectra unveiling an unprecedent $1(-1 /+1)$ asymmetric catalysis. ${ }^{26}$ This method has been extended to the less favorable six-membered ring oxidative cyclization to access 2 -acyl-chromane derivatives. ${ }^{26}$ In a similar fashion, highly enantioenriched 2-acyl tetrahydrofuran derivatives 14 have been obtained via cycloetherification of corresponding $\delta$-hydroxyketones 13 using cumene hydroperoxide (CHP) as stoichiometric co-oxidant (Scheme $8 b) .^{27}$
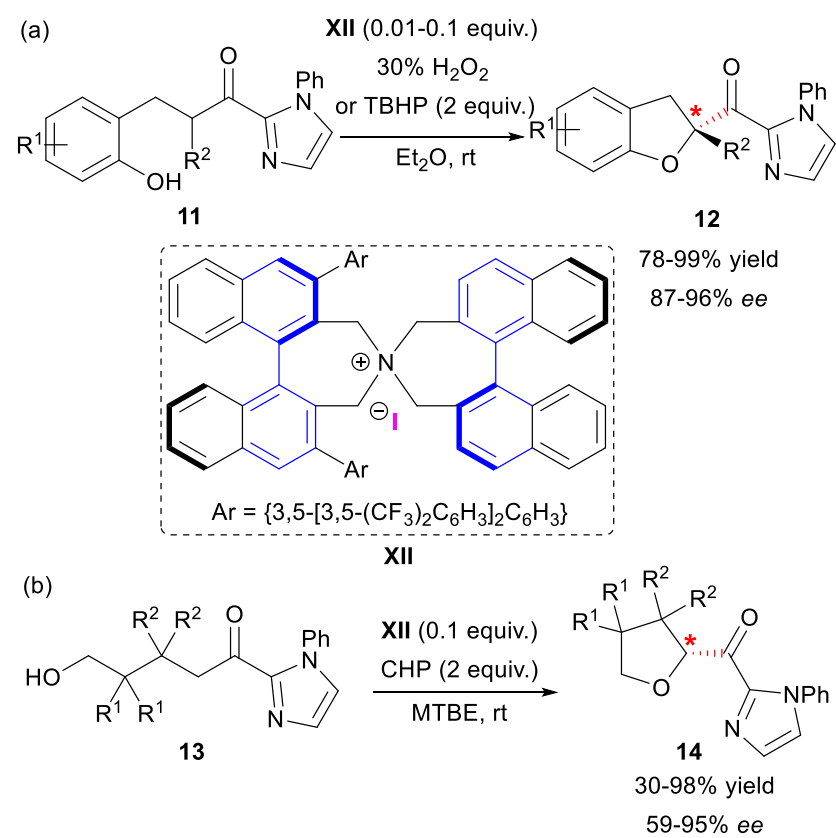

Scheme 8 In situ generated chiral hypoiodite-catalyzed oxidative cyclization. 


\section{3. $\alpha$-Fluorination}

A chiral iodoarene-catalyzed asymmetric nucleophilic $\alpha$ fluorination of indanone-based $\beta$-ketoesters 15 has been initially reported by Kita, Shibata et al. using $C_{2}$-symmetric axially chiral precatalyst $\mathbf{V I}$ in combination with $\mathrm{mCPBA}$ as a stoichiometric oxidant and $n \mathrm{HF} \cdot$ pyridine as fluorine source (Scheme 9). ${ }^{28}$ Moderate enantioselectivity was obtained with methyl ester 15a, whereas sterically demanding adamantyl ester 15b afforded a higher enantiomeric excess (56\% ee vs. $25 \%$ ee). A breakthrough has been very recently accomplished by Rueping et al. by using chiral iodoarenes derived from 2iodoresorcinol and lactate scaffolds with triethylamine pentahydrofluoride as the fluorine source. ${ }^{29}$ More particularly, the authors developed the flexible $\mathrm{C}_{2}$-symmetrical menthol ester derivative XIII bearing a 3,5-bis(trifluoromethyl)-phenyl group para to the iodine providing fluorinated product 16 in good yields and with high enantioselectivities. Chiral iodoresorcinol derivative lacking $\mathrm{C}_{2}$-symmetry proved to be inferior. In parallel to Rueping's work, Lu, Zheng et al. have designed a novel planar chiral iodoarene XIV based on [2,2]paracyclophane and bearing a benzoyl group as potentially coordinating function of the ortho- iodine atom. Triethylamine trihydrofluoride (TEA.3HF) was selected as fluorine source. It is worth mentioning that a positive effect on the enantioselectivity was observed with the introduction of methoxy-groups on the phenyl ring of the benzoyl. ${ }^{30}$ In line with experimental observations, these three research groups assumed in situ generation of $\mathrm{Ar}^{*}-\mathrm{I}-\mathrm{F}_{2}$ from $\mathrm{Ar}^{*}-\mathrm{I}, \mathrm{MCPBA}$ and $\mathrm{HF}$ along with activation of this key chiral reactive species by an excess of HF. Based on DFT calculations, Rueping et al. proposed a mechanism with formation of an $\mathrm{O}$-bonded hypervalent iodine intermediate 17. ${ }^{29}$ Migration of the aryl iodonium from the $O$-ester enolate to the $\alpha-C$ atom would lead to intermediate 18 via transition state $\mathbf{1 7} \rightarrow \mathbf{1 8}$ and yield the $\alpha$-fluorinated product through reductive elimination. The competitive $\alpha$ hydroxylation of the carbonyl compounds is responsible of the moderate yield. Transition state $\mathbf{1 7} \rightarrow \mathbf{1 8}$ is anticipated to be the enantioselectivity-determining step. According to it, formation of $(R)$-18 is favored as transition state (pro-R) $\mathbf{1 7} \rightarrow \mathbf{1 8}$ was found $1.4 \mathrm{kcal} / \mathrm{mol}$ lower in energy than transition state (pro- $S$ ) $17 \rightarrow$ 18. Reductive elimination would afford $\alpha$-fluorinated $(R)$ product 16, which is in agreement with experimentally observed stereochemistry. Unfortunately, the authors did not provide any calculations concerning the energy of a transition state corresponding to a pseudo-intramolecular $\mathrm{S}_{\mathrm{N}} 2^{\prime}$ type reductive elimination of an $\mathrm{O}$-iodonium keto-enolate, as it was proposed for the $\alpha$-oxytosylation of carbonyl compounds by Legault et al. (scheme 1, intermediate $3, \mathrm{~L}=\mathrm{F}$ ). ${ }^{20}$

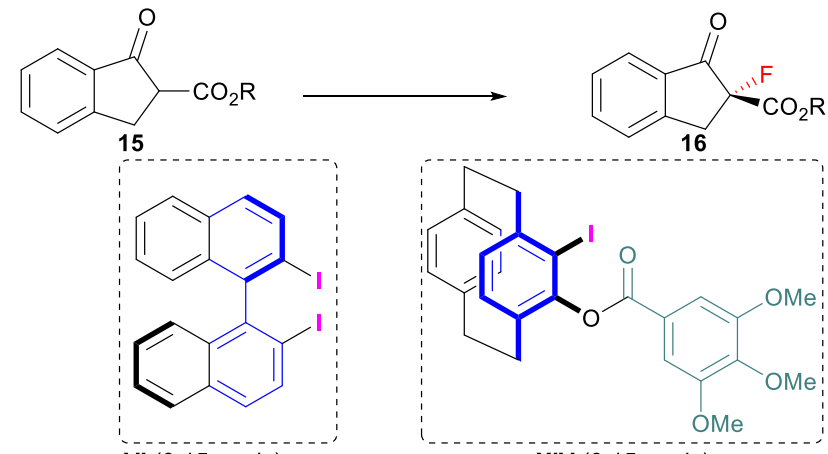

XIV (0.15 equiv)

$\mathrm{Et}_{3} \mathrm{~N} .3 \mathrm{HF}$ ( 5 equiv.)

nHF.pyridine (10 equiv.)

$m$-CPBA ( 1.3 equiv.)

toluene, rt

16a $\mathrm{R}=\mathrm{Me}, 71 \%$ yield, $25 \%$ ee 16b $\mathrm{R}=\mathrm{Ad}, 41 \%$ yield, $56 \%$ ee

$$
\mathrm{CH}_{2} \mathrm{Cl}_{2} \text {, rt }
$$

16a $\mathrm{R}=\mathrm{Me}, 71 \%$ yield, $58 \%$ ee 16b $\mathrm{R}=\mathrm{Ad}, 38 \%$ yield, $92 \%$ ee 16d $R=t-B u, 45 \%, 91 \%$ ee

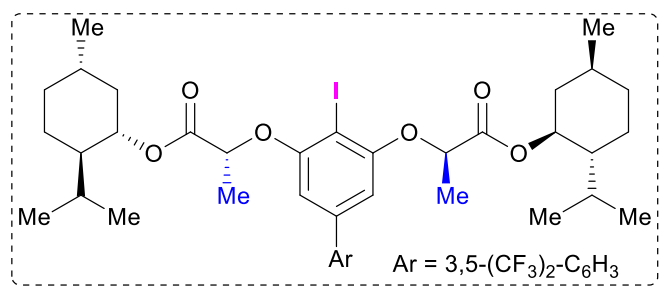

XIII ( 0.1 equiv.)

$\mathrm{Et}_{3} \mathrm{~N} .5 \mathrm{HF}$ ( 5 equiv.)

$m$-CPBA (1.5 equiv.)

$\mathrm{CHCl}_{3}, \mathrm{rt}$

16c R $=\mathrm{Et}, 67 \%$ yield, $90 \%$ ee

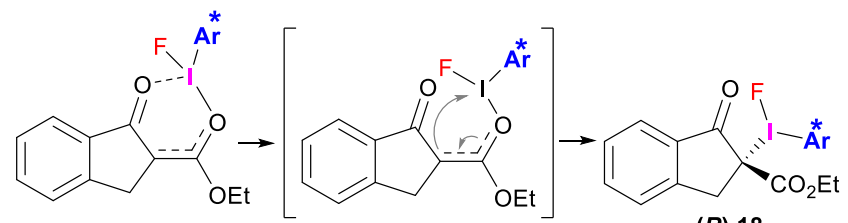

$17\left(-27.2 \mathrm{kcal} \mathrm{mol}^{-1}\right) \quad$ (pro-R) 17-18 $\left(-9.7 \mathrm{kcal}^{\left.-\mathrm{mol}^{-1}\right)}\right.$ (pro-s) 17-18 (-11.1 kcal.mol $\left.{ }^{-1}\right)$ enantioselectivity determining step

(R) 18

$\left(-42.3 \mathrm{kcal}^{\mathrm{mol}}{ }^{-1}\right)$

(R) $16 \mathrm{c}$

$\left(-90.9\right.$ kcal. $\left.\mathrm{mol}^{-1}\right)$

Scheme $9 \alpha$-fluorination of indanone derivatives.

\subsection{Spirocyclization through $\mathrm{C}\left(s \mathrm{p}^{2}\right)-\mathrm{C}\left(\mathrm{sp}^{3}\right)$ coupling}

Although most hypervalent iodine (III) catalyzed-asymmetric oxidative coupling protocols enable the formation of $\mathrm{C}-\mathrm{X}$ bonds $(\mathrm{X}=\mathrm{O}, \mathrm{N}, \mathrm{F})$, an elegant intramolecular $\mathrm{C}\left(\mathrm{sp}^{2}\right)-\mathrm{C}\left(\mathrm{sp}^{3}\right)$ coupling reaction of $N^{1}, N^{3}$-diphenylmalonamide 19 has been achieved by Gong et al. in 2014 (Scheme 10). ${ }^{31}$ This transformation provided access to biologically important spirobisoxindoles $\mathbf{2 0}$ with a quaternary carbon stereogenic center in good yields and with good enantiomeric excesses. The best reaction conditions involved the use of ethaneperoxoic acid $\left(\mathrm{MeCO}_{3} \mathrm{H}\right)$ as the stoichiometric oxidant in nitromethane with $\mathrm{C}_{2}$-symmetric iodoresorcinol derivative $\mathbf{X V}$ as precatalyst. Notably, the tertiary proline amide on $\mathbf{X V}$ (at the end of the resorcinol arms) played key role in inducing the highest enantioselectivities compared to secondary amides, carboxylic acids or esters. 
Moreover, the addition of 2 equivalents of trifluoroacetic acid enhanced the catalytic performances in terms of both yield and enantioselectivity. To shed light on the reaction mechanism and the origin of the stereoselectivities, computational studies have been undertaken by Sunoj et al. ${ }^{32}$ Based on DFT calculations, the authors proposed a mechanism during which the in situ generation of the key chiral hypervalent iodine intermediate $\mathrm{Ar}^{*}-\mathrm{I}(\mathrm{TFA})_{2}$ was the initial step. Ligand exchange with the nitrogen atom of incoming dianilide substrate 19 followed by pseudo-intramolecular deprotonation of $\mathbf{2 1}$ by the leaving trifluoroacetate anion generated $O$-iodonium enolate 22 . The ensuing $O$ to $C$-aryliodonium migration leads to $C$-iodonium enolate $\mathbf{2 3}$ with a lower energy. Intramolecular Friedel-Craft reaction assisted by the trifluoroacetate anion allowed the formation of the first oxindole ring $\mathbf{2 4}$ with concomitant regeneration of precatalyst $\mathbf{X V}$. The second ring formation would be enabled by a similar reaction sequence yielding spirobisoxindole 19. It is worth mentioning that direct ring closings from $O$-iodonium $\mathbf{2 2}$ and $\mathbf{2 6}$ have been calculated to be of higher energy, indicating that formation of $C$-iodonium enolates 23 and $\mathbf{2 7}$ might occur. As chiral information of intermediate $\mathbf{2 4}$ is lost during the second catalytic cycle, stereoselectivity of the reaction is solely governed during the second ring closure. More precisely, the 1,3-migration of the chiral aryl iodonium from $O$ to $C$-enolate $(\mathbf{2 6} \rightarrow \mathbf{2 7})$ is the stereocontrolling step of the reaction. The transition states leading to $(S)$-product 19 were found to be $2.1 \mathrm{kcal} / \mathrm{mol}$ lower than those leading to the $(R)$-product, which is in good agreement with the experimentally obtained enantioselectivity. Noteworthily, in the preferred conformer of key chiral hypervalent iodine intermediate $\left.\mathrm{Ar}^{*}-\mathrm{I}_{(\mathrm{TFA}}\right)_{2}$, resorcinol arms were found to organize in a $\mathrm{C}_{2}$-symmetric helical assembly with a right-handed $P$ helicity in which the two trifluorooacetate ligands form a network of nonconvalent interactions with the chiral arms.
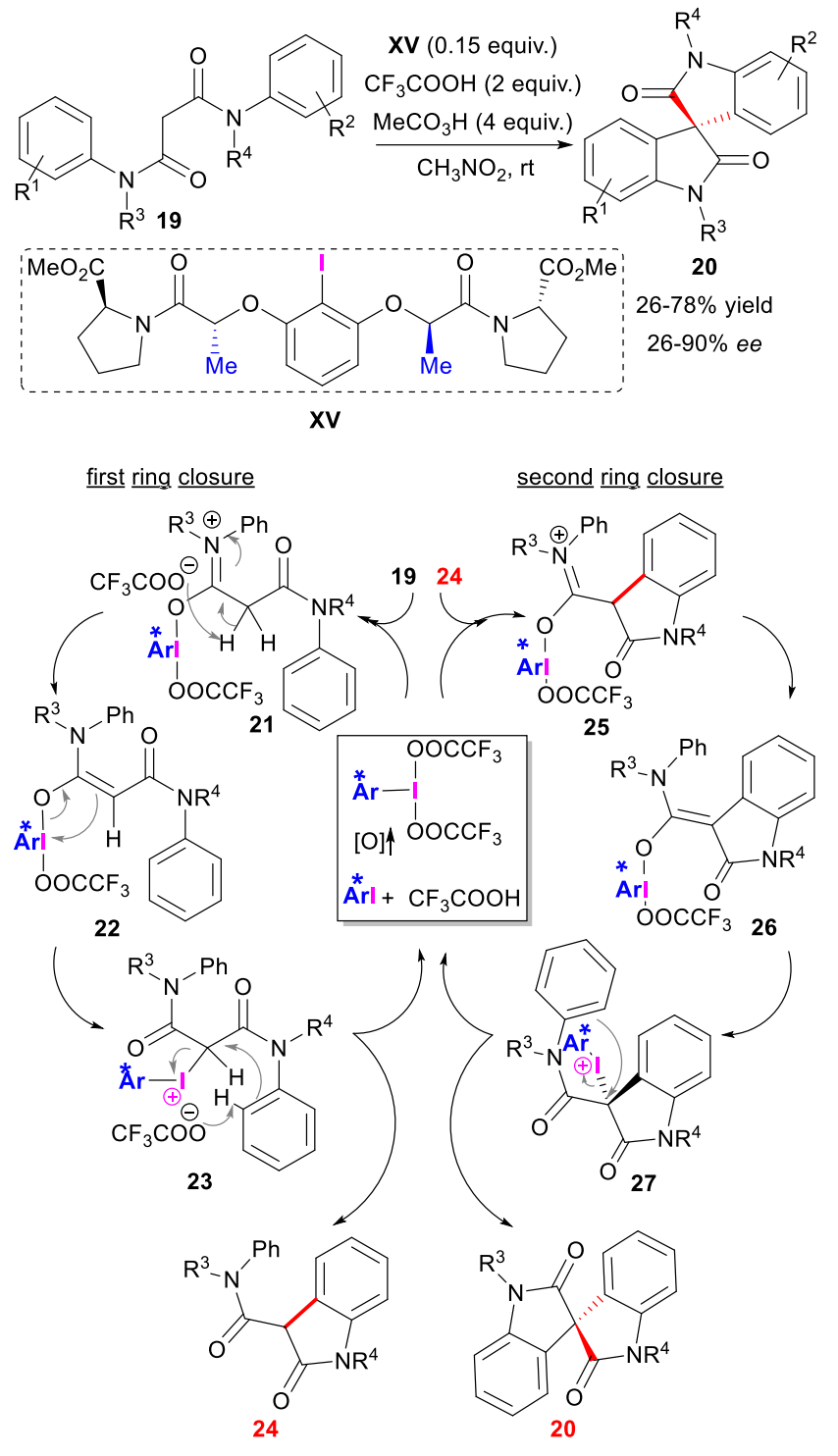

Scheme 10 Spirocyclisation through two sequential C-C cyclizing couplings.

\section{Dearomatization of phenol derivatives}

The asymmetric dearomatization of phenol derivatives is of particular interest for the synthetic community as it can be a pivotal step in the total synthesis of natural products. Chiral hypervalent organoiodines reagents are organo-oxidants of choice to perform this transformation enantioselectively. ${ }^{33}$ They can be generated in situ from a catalytic amount of chiral iodoarene and mCPBA as a stoichiometric oxidant. Ligand exchange between the phenol and in situ generated iodine (III) would furnish phenoxy- $\lambda^{3}$-chiral aryliodarene 28 (Figure 3 ). Subsequently, two mechanisms are generally considered: direct attack of the nucleophile via a $\mathrm{S}_{\mathrm{N}} 2$ '-type substitution (associative path) or formation of phenoxenium ion $\mathbf{2 9}$, which is trapped by the nucleophile (dissociative path). The key feature to successively achieve asymmetric induction is to find reaction conditions (catalyst, solvent, additive...) capable of favorizing the associative mechanism. 


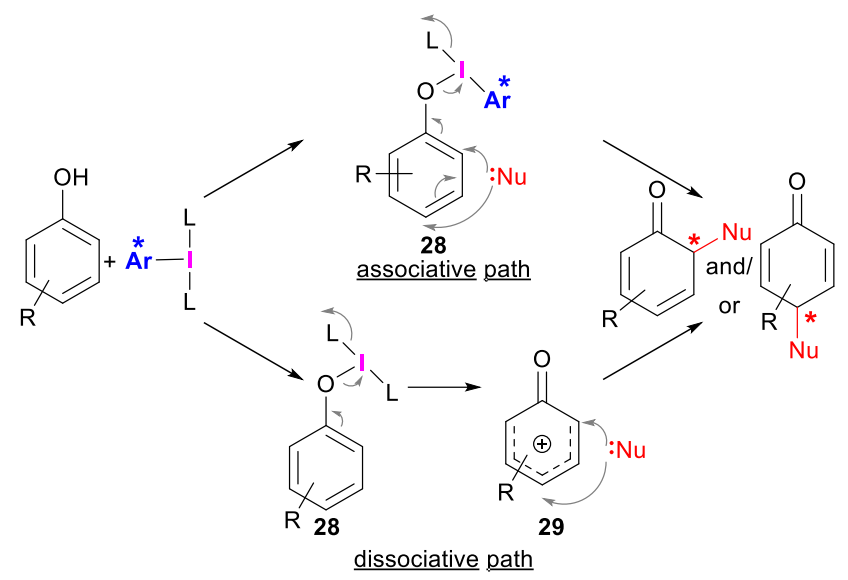

Figure 3 Proposed mechanisms for organoiodane-mediated phenol dearomatization.

\subsection{Intramolecular transformations}

The intramolecular dearomatization of phenol derivatives provides a straightforward way to spirocyclic compounds. The asymmetric dearomatization of propanoic acid substituted 1naphthol $\mathbf{3 0}$ to spirolactone $\mathbf{3 1}$ has been intensively studied as a model reaction for the design of new aryliodine precatalysts. Indeed, precatalysts which exhibits either $\mathrm{C}_{1}$ - or $\mathrm{C}_{2}$-symmetry and central or axial- chirality have been developed to achieve the highest enantioselectivity (Scheme 11).

In 2017, Nachtsheim and Pericàs et al. reported a new $\mathrm{C}_{1-}$ symmetric triazole-based chiral iodoarenes (XVI). This catalyst was prepared thanks to an enzymatic kinetic resolution followed by a copper-catalyzed azide-alkyne cycloaddition and provided spirolactone 31a with moderate yield and good enantioselectivity.

Ishihara et al. developed conformationally flexible $C_{2}-$ symmetric chiral iodoarenes $\mathbf{X V I I}$ derived from lactate as a source of chirality affording spirolactone 31a in good yield and with high enantioselectivity $\left(55 \%, 92 \%\right.$ ee).${ }^{34}$ More recently, an enhancement of the yield and the enantioselectivity was achieved in the same group by using catalyst XVIII derived from chiral amino-alcohol at low loadings. ${ }^{35} \mathrm{X}$-ray and NMR analyses of in situ generated organoiodine(III) species from XVIII showed that intramolecular hydrogen-bonding interactions between acidic amido protons and ligands of iodine(III) allowed the construction of a suitable chiral environment around the iodine(III) center. ${ }^{36}$

An original $\mathrm{C}_{2}$-symmetric iodoarene $\mathbf{X I X}$ with central chirality based on the rigid all-carbon anti-dimethanoanthracene framework displayed lower activity and selectivity. ${ }^{37}$ However, a rigid $\mathrm{C}_{2}$-symmetric aryiliodine $\mathbf{V b}$ based on the axially chiral spirobiindane scaffold has been disclosed by Kita et al. in 2008 . Cyclized product 31a has been obtained in good enantioselectivity with this catalyst. ${ }^{38}$ An enhancement of the enantioselectivity was observed with Vc via introduction of ethyl-substituents at the ortho-positions of the iodine atoms, presumably thanks to an extension of surroundings around the reactive sites. ${ }^{39}$

In 2016, Masson et al. explored axially chiral 1,1'binaphtalene framework and derivatives for the development of novel chiral iodoarenes prepared from commercially available

(R)-1,1'-binaphtyl-2,2'-diamine

(BINAM)..$^{40}$ Spirolactone 31a was obtained with little enantiomeric excess with $\mathrm{H}_{8}$-BINI XXa. Introduction of 1-naphtyl substituents at the 3,3'-positions (XXX) exerted a slightly beneficial effect on enantioselectivity. Interestingly, a non $\mathrm{C}_{2}$-symmetric iodoarene precatalyst (XXI) harboring an amide group at the 2 '-position afforded spirolactone 31a in poor enantioselectivity but with an inversion of configuration. By analogy with Ishihara and Wirth's catalysts, additional non-covalent interactions between the amide group and the iodine (III) center or its ligands may be responsible of this reversal through a different transition state. Kita et al. designed a new type of chiral 1,1'-binaphthalene (XXII) substituted with the iodine atoms at the $8,8^{\prime}$-positions which dramatically improved enantioselectivity, but at the price of a high catalyst loading (0.55 equiv.). ${ }^{41}$ This precatalyst bearing the iodine atoms within the major groove of the naphtalene rings has been synthesized by a nickel-catalyzed reductive homocoupling, enantiomeric resolution of the obtained racemic diamine on preparative HPLC with chiral stationary phase column, followed by Sandmeyer-type iodination. 

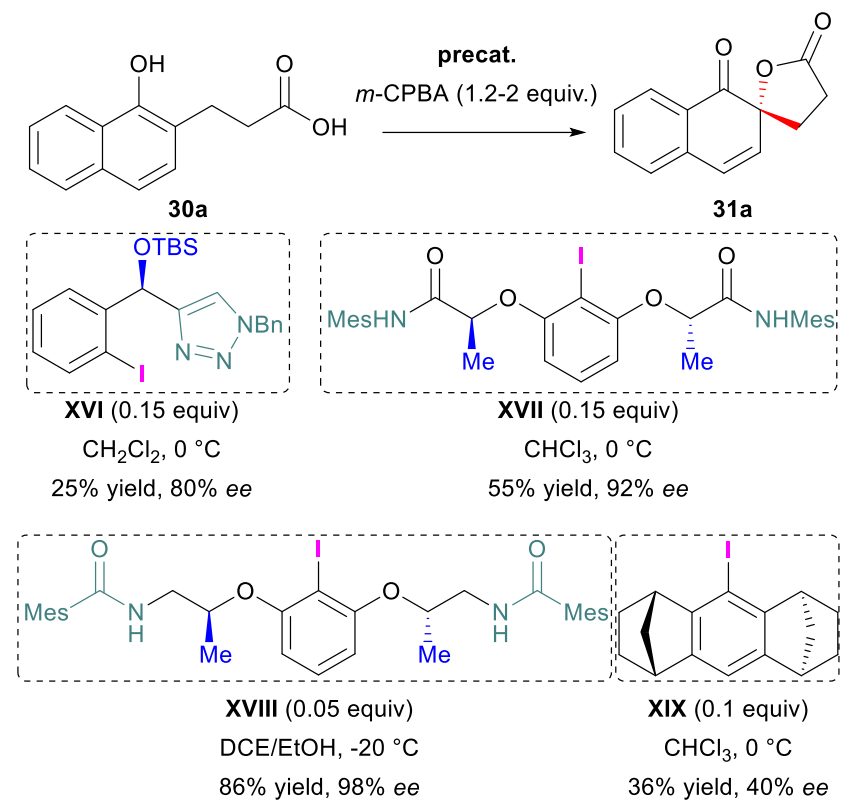

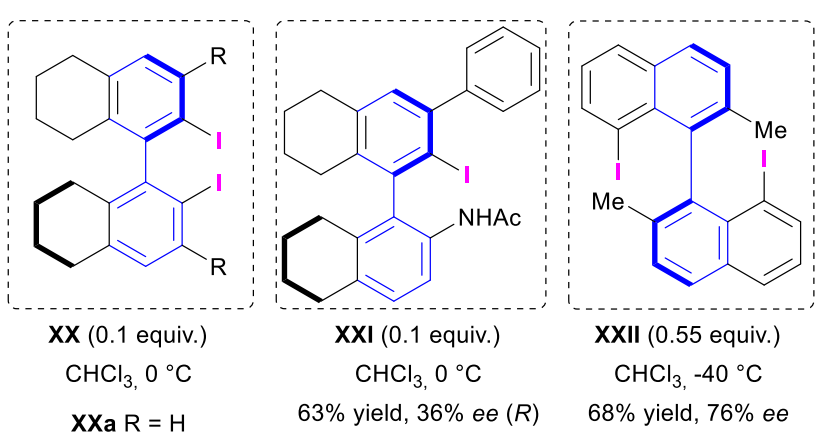

$67 \%$ yield, $22 \%$ ee $(S)$

XXb R = 1-naphtyl

$63 \%$ yield, $36 \%$ ee $(\mathrm{S})$

Scheme 11 Asymmetric dearomatization of propanoic acid substituted 1-naphthol 30a.

In 2015, Ishihara et al. reported application of their strategy of in situ generated-chiral ammonium hypoiodite from the corresponding chiral ammonium iodide and hydrogen peroxide as stoichiometric oxidant to the catalytic spirolactonization of 1-naphtol derivatives with good yields and good enantioselectivities at room temperature (Scheme 12). ${ }^{42}$ The use of hydrogen-peroxide as stoichiometric oxidant offers the advantage of generating only water as a by-product instead of mCBA, but limited the scope of the transformation to 3 or 4substituted naphtols.

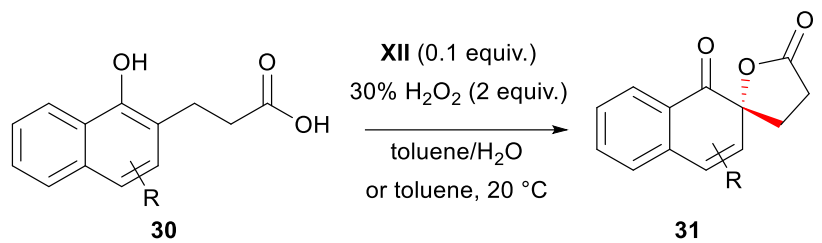

up to $90 \%$ yield, $92 \%$ ee

Scheme 12 Hypoiodite-catalyzed asymmetric dearomatization of 3 or 4 substituted 1 naphthol derivatives $\mathbf{3 0}$.

In 2013, Ishihara et al. investigated oxidative cyclizative dearomatization of phenol derivatives $\mathbf{3 2}$ by using precatalyst XVIII (Scheme 13). ${ }^{36}$ In the case of poorly reactive electrondeficient phenols, the addition of 50 equivalent of $1,1,1,3,3,3$ hexafluoroisopropanol (HFIP) was required and dramatically enhanced efficiency of the process. ${ }^{43}$ In contrast, addition of 25 equivalents of methanol was necessary in the case of highly reactive electron-rich phenols to presumably ensure a ligand exchange from acyloxyphenoxyiodine (III) 34. This protocol would slow down the racemic dissociative pathway and would favor the enantioselective associative pathway as methoxy ligand is a less good leaving group than carboxylate ligand. Under these conditions, high yields and excellent enantioselectivities were obtained in both cases. Noteworthily, this protocol has proved to be also very efficient for the spirolactonization of poorly reactive 2-naphtol derivatives ${ }^{35}$ and for dearomatization of ortho- and para-hydroquinone derivatives allowing the construction of ortho- and parabenzoquinones. ${ }^{44}$

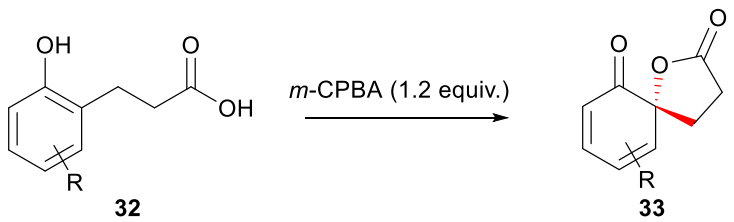

if $\mathrm{R}=\mathrm{EWG}, \mathbf{X V I I I}\left(0.05\right.$ equiv.), $\mathrm{CH}_{2} \mathrm{Cl}_{2}, \mathrm{HFIP}$ (50 equiv.), $-20^{\circ} \mathrm{C}$ if $\mathrm{R}=\mathrm{EDG}, \mathbf{X V I I I}$ ( 0.1 equiv.), $\mathrm{CH}_{2} \mathrm{Cl}_{2}, \mathrm{MeOH}$ (25 equiv.), $-10^{\circ} \mathrm{C}$ $78-88 \%$ yield, $87-98 \%$ ee

Proposed explanation for the role of $\mathrm{MeOH}$ if $\mathrm{R}=\mathrm{EDG}$ :

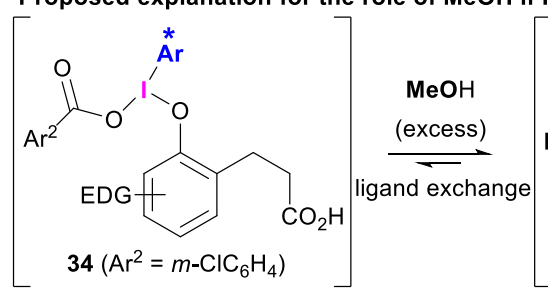

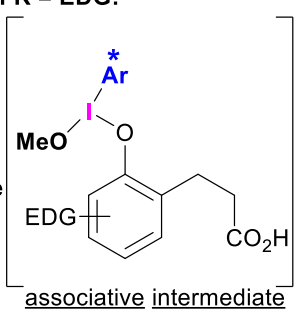

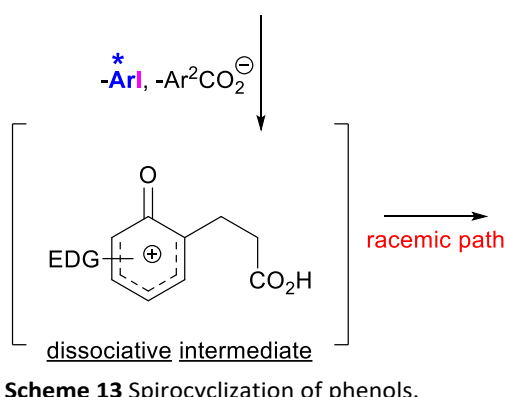

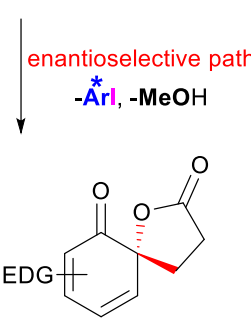


In 2017, Ciufolini et al. described the hypervalent-iodine catalyzed enantioselective oxidative cycloetherification reaction of naphtolic alcohols $\mathbf{3 5}$ (Scheme 14). ${ }^{45}$ The design of a new precatalyst was required as these substrates are less reactive than the corresponding carboxylic acids. In this context, a variant of the Ishihara's catalyst was examined. Considering that intramolecular $\mathrm{H}$-bonding between the secondary amide $\mathrm{NH}$ group and the ligand of the iodine III is the key feature in these type of catalysts, the authors relocated the stereogenic center closer to the iodine atom. As a result, XXIII provided spiro compounds $\mathbf{3 6}$ in high yields and with excellent enantioselectivities. This procedure has been extended to oxidative cyclization of naphtolic sulfonamides with good enantioselectivity.<smiles>[R]c1cc(CCCO)c(O)c2ccccc12</smiles>

35

$\mathrm{R}=\mathrm{H}, \mathrm{Cl}, \mathrm{Br}, \mathrm{Ar}, \mathrm{Me}$, ester

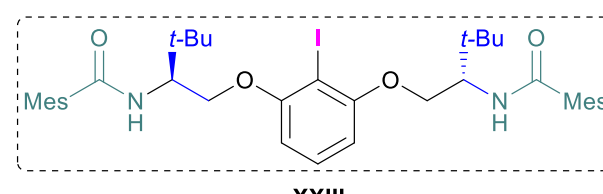

XXIII<smiles>[R]C1=C[C@]2(CCCO2)C(=O)c2ccccc21</smiles>

36

$15-79 \%$ yield $75-93 \%$ ee relocating the stereogenic center closer to the $\mathrm{H}$-bound donor

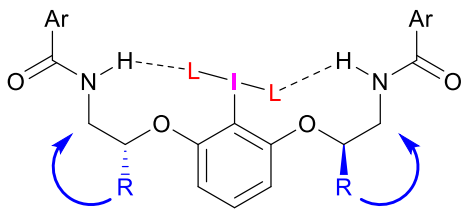

Scheme 14 A modification of Ishihara's type catalysts for the spirocyclization of naphtolic alcohols.

In their continuous efforts to exploit chiral hypervalent iodine catalysis for the creation of C-C bonds, Gong et al. have disclosed a highly enantioselective dearomatizative spirocyclization of 1-hydroxy- $N$-aryl-2-naphthamide derivatives 37, providing access to spiroxindoles derivatives $\mathbf{3 8}$ with good yields and enantioselectivity. ${ }^{46}$ Precatalyst XVII was the aryliodine of choice for this transformation in the presence of mCPBA as a stoichiometric oxidant. The reaction would proceed through the formation of phenoxy- $\lambda^{3}$-iodine species 39 , which would undergo intramolecular $\mathrm{S}_{\mathrm{N}} 2^{\prime}$-like Friedel-Crafts substitution. The addition of a mixture of trifluoroethanol and water was critical for the improvement of both yields and enantioselectivity (Scheme 15). ${ }^{43}$ As previously suggested by Ishihara et al., these additives would facilitate ligand exchanges to favor the enantioselective associative pathway over the racemic dissociative pathway.<smiles>[R]c1cc(C(=O)N([R])c2ccccc2)c(O)c2ccccc12</smiles>

$\mathrm{R}^{1}=\mathrm{H}, \mathrm{Br} ; \mathrm{R}^{2}=\mathrm{Me}, \mathrm{Ph}$

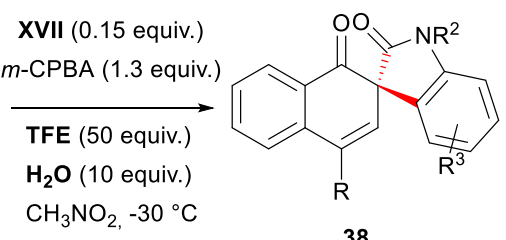<smiles>[Y][IH2]Oc1c(C(=O)N([R])c2ccccc2)cc([R])c2ccccc12</smiles>

$$
X=\mathrm{CF}_{3} \mathrm{CH}_{2} \mathrm{O} \text { or } \mathrm{OH}
$$

Scheme 15 Oxidative $\mathrm{C}-\mathrm{C}$ coupling-enabled naphtols dearomatization.

\subsection{Intermolecular transformations}

Asymmetric intermolecular phenol dearomatization is a highly challenging task as this transformation can easily proceed through a racemic dissociative pathway. ${ }^{47}$ Quideau et al. have pioneered catalytic enantioselective hydroxylative phenol dearomatization. ${ }^{48}$ Axially chiral monoiodobinaphtyl XXIV was used in the presence of a stoichiometric amount of MCPBA to convert 2-methylnaphtol $\mathbf{4 0}$ into epoxy ortho-quinol $\mathbf{4 1}$ in good yield and with moderate enantioselectivity. Unfortunately, epoxidation of the ortho-quinol by the stoichiometric oxidant cannot be avoided. Secondary $1 \cdots \mathrm{O}$ interactions between the iodine (III) atom and the methoxy group and/or the carboxylic acid function were assumed, but the real nature $\left(\lambda^{3}\right.$ or $\left.\lambda^{5}\right)$ of reacting hypervalent iodine involved in this transformation could not be determined with certainty.
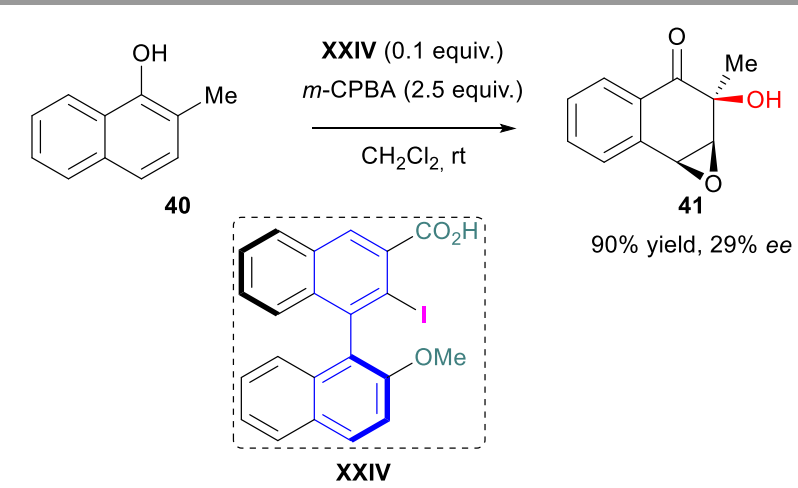

$90 \%$ yield, $29 \%$ ee

Scheme 16 Intermolecular naphtol dearomatization.

Hypervalent

iodine-catalyzed

intermolecular dearomatization of phenol can also enable introduction of a nucleophile at the para position of the hydroxyl group. Designing an enantioselective version of such a reaction seems to be even more arduous, as the creation of the stereogenic center occurs quite far away from the chiral environment (namely the phenoxy- $\lambda^{3}$-chiral aryliodane). To address this challenge, Harned et al. designed $\mathrm{C}_{1}$-symmetric chiral iodine $\mathbf{X X V}$ derived from 8-iodotetralone and tartaric acid for the 4- 
hydroxylation of phenols $\mathbf{4 2}$ in the presence of mCPBA as the stoichiometric oxidant (Scheme 17). ${ }^{49}$ The resulting 2,5cyclohexanedienones $\mathbf{4 3}$ were obtained in moderate yields and with enantioselectivity up to $60 \%$ ee. In 2017, Muñiz et al. proposed the use of $\mathrm{C}_{2}$-symmetric lactic amide XXVI (Ishihara's type precatalyst), which exhibited slightly lower stereoselectivities for the same transformation..$^{50}$ Very recently, Maruoka et al. have developed $\mathrm{C}_{1}$-symmetric indanol-based chiral organoiodine XXVII allowing the access to 2,5cyclohexadienones $\mathbf{4 3}$ with the highest level of enantioselectivity reported to date. ${ }^{51}$<smiles>[R]c1cc(C)ccc1O</smiles>

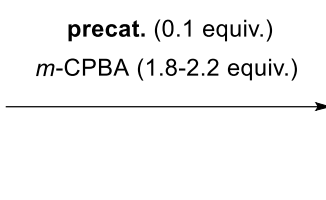

42

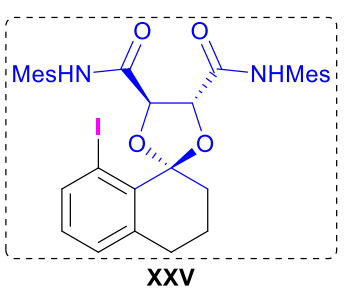

$\mathrm{CH}_{3} \mathrm{CN} / \mathrm{H}_{2} \mathrm{O}(9: 1)$, rt up to $79 \%$ yield, $60 \%$ ee

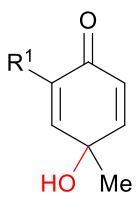

43

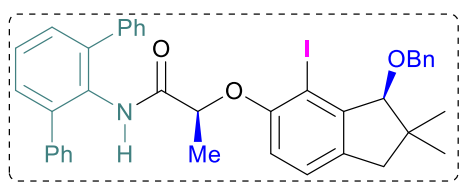

XXVII

butanone $/ \mathrm{H}_{2} \mathrm{O}(2: 1), 0{ }^{\circ} \mathrm{C}$ up to $73 \%$ yield, $84 \%$ ee

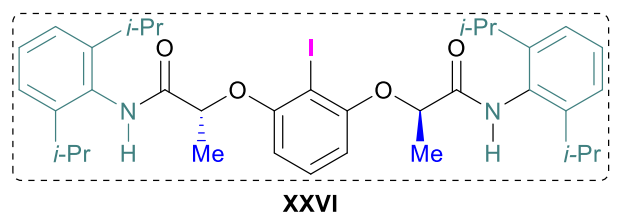

$\mathrm{CH}_{3} \mathrm{CN} / \mathrm{H}_{2} \mathrm{O} 9: 1$ or $\mathrm{CH}_{3} \mathrm{CN} / \mathrm{TFE} / \mathrm{H}_{2} \mathrm{O}(8: 1: 1),-5{ }^{\circ} \mathrm{C}$ up to $96 \%$ yield, $50 \%$ ee

Scheme 17 4-hydroxylation of phenols.

\section{Functionalization of alkenes}

Hypervalent iodine-mediated asymmetric difunctionalization of alkenes is a useful strategy for the preparation of polysubsituted molecules with the creation of one or two vicinal stereogenic centers from either two identical or different nucleophiles. ${ }^{52}$

\subsection{Intramolecular version}

In its intramolecular version, such a reaction enables the construction of polysubstituted heterocycles in one step.

4.1.1. Lactonization. The first example of a catalytic difunctionalization of alkenes mediated by chiral hypervalent iodine has been reported by Fujita et al. In their original reports, the authors disclosed an oxidative double cyclization of 2-(4hydroxybut-1-enyl)benzoates 44 (Scheme 18). ${ }^{53}$ By using precatalyst XXVIII, $\mathrm{mCPBA}$ as the terminal oxidant and trifluoroacetic acid as an activator, the dihydrofuran-fused isochromanones 45 were obtained in moderate yields and enantioselectivities up to $91 \%$ ee. The reaction would proceed through the diastereofacial attack of the chiral iodane on the double bond to form iodonium 46. The latter would undergo nucleophilic substitutions by the internal hydroxy and carboxymethyl groups with inversion of configuration. The competitive direct oxidation of the double bound by MCPBA is responsible of the moderate yields.
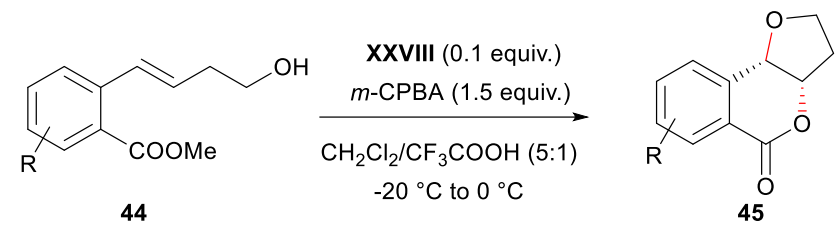
$25-64 \%$ yield

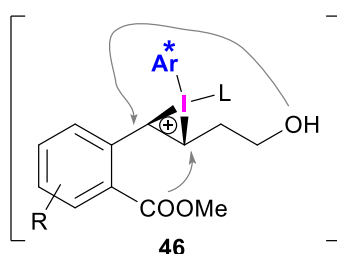
$55-91 \%$ ee

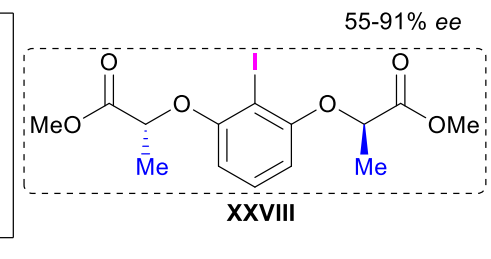

Scheme 18 Oxidative double cyclization.

The background oxidation with mCPBA has been well identified by the same research group in the hypervalent-iodine catalyzed trifluoroacetoxylactonisation of ortho-alk-1enylbenzoates 47 (Scheme 19). ${ }^{54}$ In this process, the catalytic oxidation mediated by chiral hypervalent iodine(III) species gives enantioenriched syn-products $48 \mathrm{a}$ and $48 \mathrm{~b}$ via ring opening of iodonium 49 , while the direct oxidation with mCPBA delivers racemic anti-products $48 \mathrm{c}$ and $48 \mathrm{~d}$ via opening of epoxide 50. Good 6-endo regioselectivity and high enantioselectivity were obtained on cis-isochromanone 48a with precatalyst XXIX. Unfortunately, the competitive background oxidation with mCPBA of the electron-rich internal double bound significantly affected the yield. 


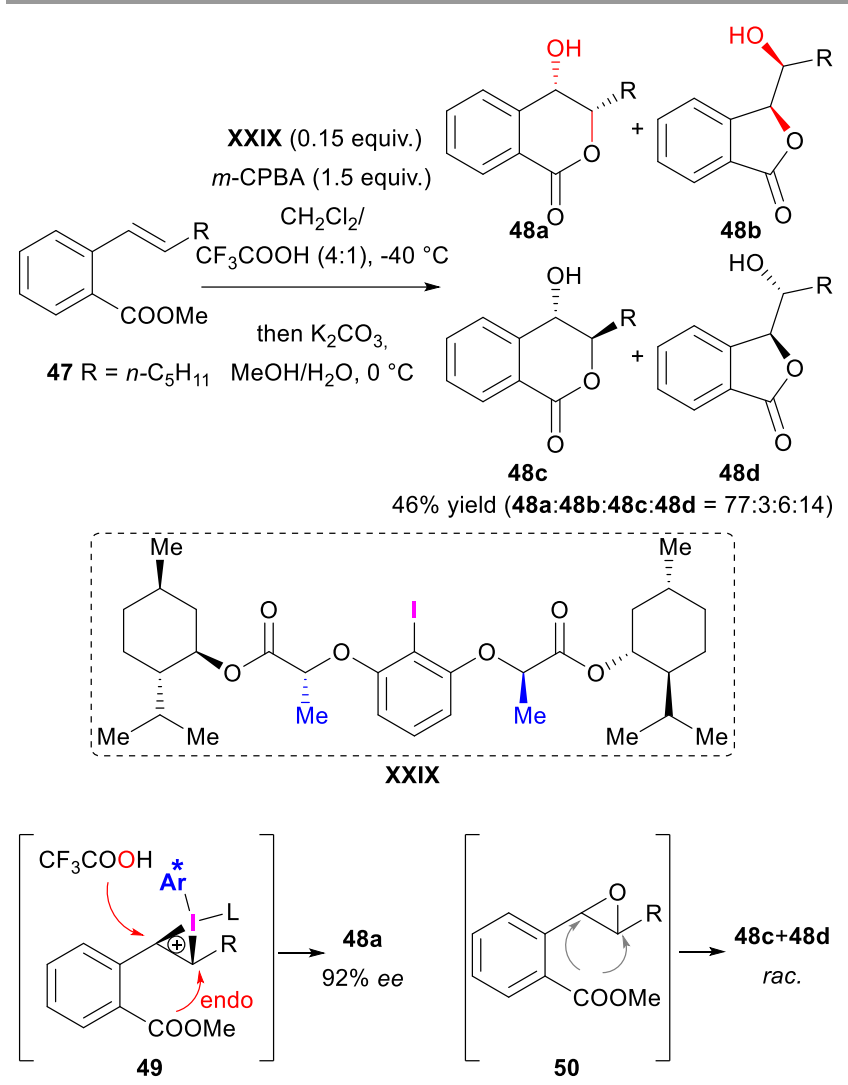

Scheme 19 Oxy-lactonization of ortho-alk-1-enylbenzoates 47.

In 2017, Masson et al. reported a tosyloxy- and phosphoryloxy-lactonization of flexible 4-pentenoic acid derivatives $\mathbf{5 1}$ (Scheme 20). ${ }^{55}$ By using XVII and a stoichiometric amount of mCPBA in the presence of sulfonic or phosphoric acid, excellent exo selectivity was observed, providing access to valuable $y$-butyrolactones $\mathbf{5 2}$ with good yields and good enantioselectivities. To minimize competitive direct oxidation of the terminal double bound by $\mathrm{mCPBA}$, the reactions needs to be carried out at $0{ }^{\circ} \mathrm{C}$. Addition of 2,2,2-trifluoroethanol (TFE) as a cosolvent was required to improve the yield of the phosphoryloxycyclization. ${ }^{43}$ From a mechanistic point of view, the authors proposed in situ generation of intermediate $\mathbf{5 3}$ from precatalyst XVII, mCPBA and the acid. Electrophilic attack of the double bound by this chiral $\lambda^{3}$-aryliodane would furnish chiral aryliodonium 54. Intramolecular nucleophilic attack of the carboxy group with exo selectivity followed by substitution of the iodonium with the sulfonyl or phosphoryl group would afford y-lactone 52

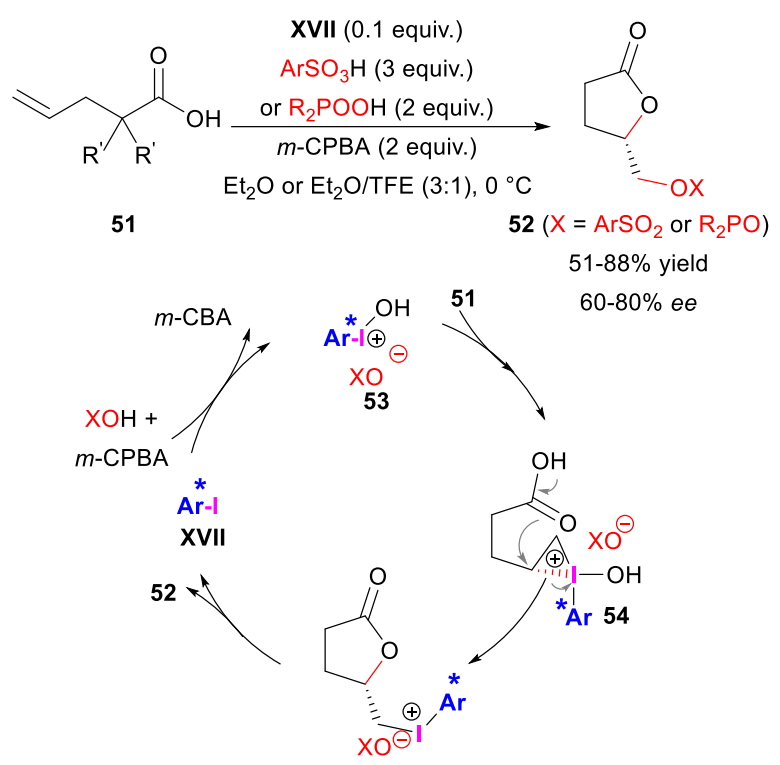

Scheme 20 Tosyloxy- and phosphoryloxy-lactonization of flexible 4-pentenoic acid $\mathbf{5 1 .}$

The hypervalent iodine catalyzed fluorolactonization of ortho-alk-1-enylbenzoates $\mathbf{5 5}$ has been investigated by Jacobsen et al. (Scheme 21). ${ }^{56}$ This method used HF.pyridine as a nucleophilic fluoride source with chiral precatalyst $\mathbf{X X X}$ and mCPBA, affording 3-alkyl-4-fluoroisichromanones $\mathbf{5 6}$ as a single syn diastereoisomer with moderate yields and high enantioselectivities. Noteworthily, the obtained regioselectivity (formation of the 6-membered ring) is complementary to that established in asymmetric fluorolactonization with an electrophilic fluoride source. This regioselectivity is explained by activation of the alkene by hypervalent iodine followed by addition of the fluoride anion onto the benzylic carbon, furnishing anti-vicinal fluoroiodonium intermediate 57 . The latter would undergo intramolecular nucleophilic substitution by the carboxymethyl group with inversion of configuration providing syn-diastereoisomer 56. 
<smiles>[M]OC(=O)C1=C(/C=C/C)C=C[R1]C=C1</smiles>

55

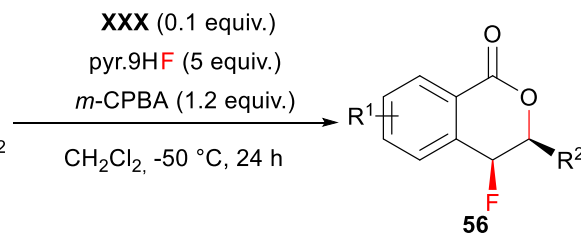

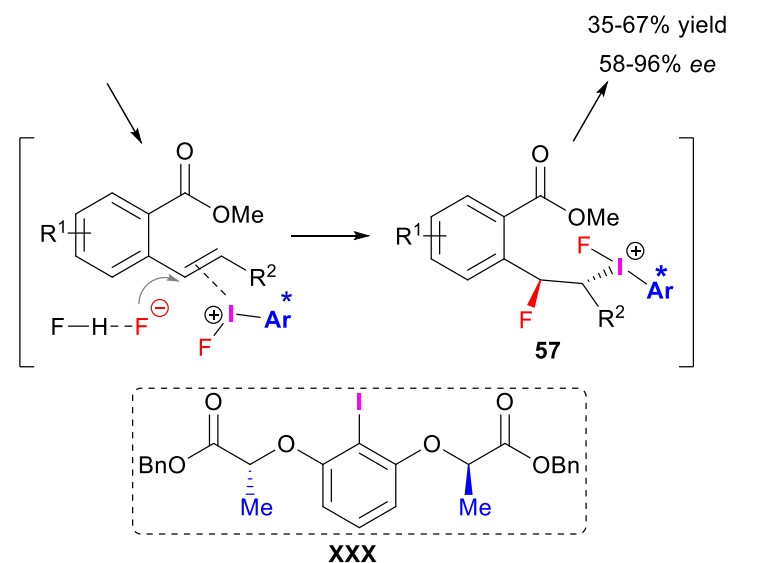

Scheme 21 Hypervalent iodine catalysed asymmetric 6-endo-type-fluorolactonization.

4.1.2. Formation of aza-heterocycles. Kita, Shibata et al. have developed the chiral aryl iodine-catalyzed intramolecular aminofluorination of $\omega$-amino-alkene $\mathbf{5 8}$ using mCPBA as the stoichiometric terminal oxidant and HF-pyridine as a nucleophilic fluoride source. ${ }^{28}$ This transformation enabled the formation of 2-fluoropiperidine $\mathbf{5 9}$ with moderate yield and enantioselectivity. According to the observed 6-endo selectivity of the process, the aminofluorination would proceed through oxidation of the sulfonamide by in situ generated $\mathrm{ArIF}_{2}$ furnishing electrophilic species $\mathbf{6 0}$. The latter would react with the double bond to release the precatalyst and concomitantly form intermediary aziridinium 61. The latter would undergo nucleophilic attack of the fluoride on the tertiary carbon to deliver endo-type cyclized product 59. The best aryliodine precatalyst evaluated was $\mathrm{C}_{2}$-symmetric axially chiral BINI VI. Strikingly, while a stoichiometric amount of chiral aryliodonium difluoride XXXIb has been reported to give $\mathbf{5 9}$ with $81 \%$ ee, ${ }^{57}$ no enantioselectivity was observed with parent aryliodine XXXIa under catalytic conditions. This observation suggests different type of mechanisms between the catalytic and stoichiometric processes. conditions A:

Arl $(0.15$ equiv.

HF.pyridine (10 equiv.)

$m$-CРBA ( 1.3 equiv.)

toluene, $\mathrm{rt}$

58

conditions B:

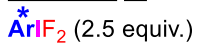

toluene, $\mathrm{rt}$

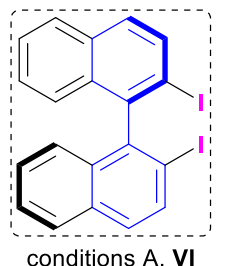

$64 \%$ yield, $59 \%$ ee

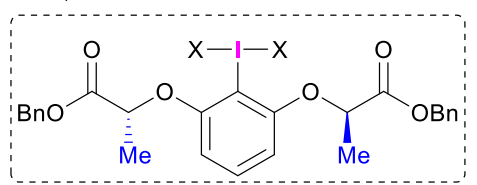

conditions A, XXXIa X = none, $43 \%$ yield, $0 \%$ ee conditions $\mathrm{B}, \mathbf{X X X I b} \mathrm{X}=\mathrm{F}, 79 \%$ yield, $81 \%$ ee

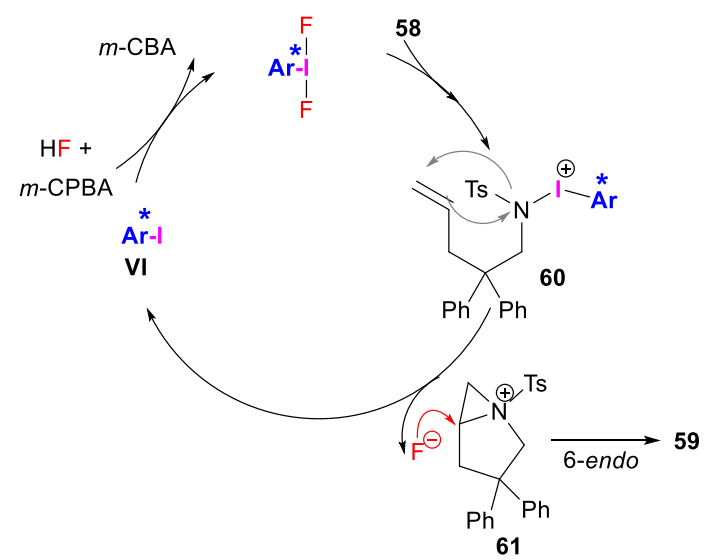

Scheme 22 Access to enantioenriched 3-aminopiperidine 59

Very recently, the highly enantioselective syn- $\beta$ fluoroaziridination of cynnamyltosylamide $\mathbf{6 2}$ has been disclosed by Jacobsen et al. (Scheme 23). ${ }^{58}$ The optimal reaction conditions are similar to those previously reported by the group for fluorolactonization (see section 4.1.1) with $\mathbf{X X X I I}$ as the precatalyst of choice. In contrast with the above fluoropiperidination reported by Kita, Shibata et al., the reaction would be initiated by oxidation of the electron-rich internal alkene by the in situ generated $\mathrm{ArIF}_{2}$ furnishing an electrophilic iodonium. Nucleophilic attack by the excess of fluoride would generate anti-vicinal fluoroiodonium 63, which would be intramolecularly trapped by the sulfonamide to give fluoroaziridines 64 . This reaction sequence accounts for the total syn-selectivity. The process is limited to electron deficient cinnamyl tosylamides since substrates lacking electron withdrawing substituents gave rise to 1,1-difluoromethylated products 66 via phenonium ion 65 (Scheme 23, R = H, EDG). Carbonyl-based protected amines (carbamates, amides) led to the formation of 1,2-oxy-fluorinated products via intramolecular nucleophilic attack of the carbonyl oxygen onto the fluoroiodonium rather than the nitrogen. 


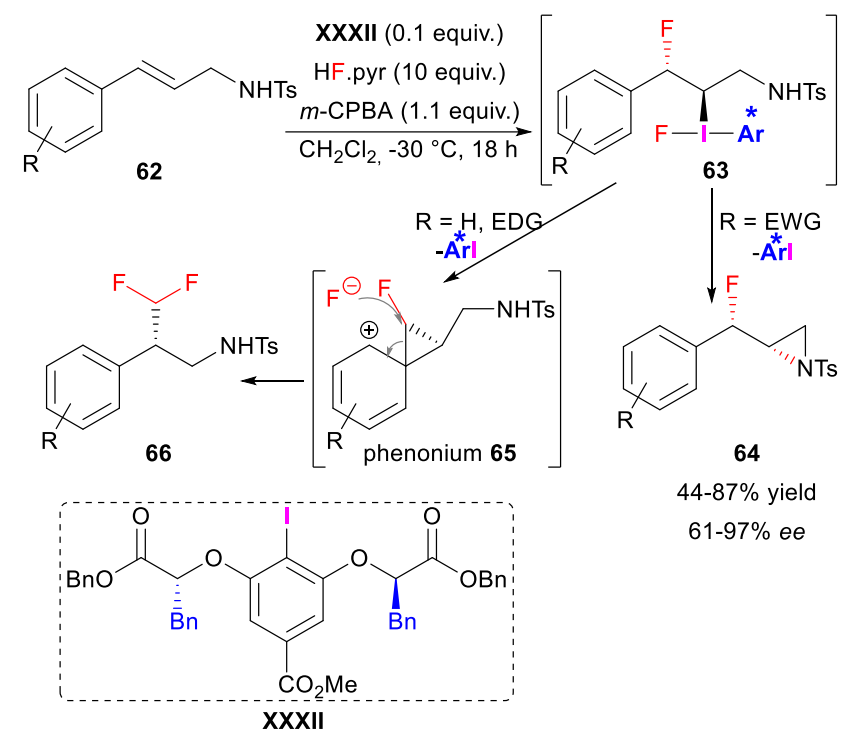

Scheme 23 Synthesis of syn- $\beta$-fluoroaziridnes and competitive rearrangement.

In 2014, Wirth et al. reported an elegant intramolecular diamination of homoallylic guanidine and diaminosulfone derivatives $67 .{ }^{59}$ In this transformation, the two attacking nucleophiles are on the substrate. Activation of the double bound by the hypervalent iodine triggered the first nucleophilic attack of one nitrogen to the more substituted carbon. $\mathrm{S}_{\mathrm{N}} 2$-type nucleophilic substitution of the resulting hypervalent moiety (intermediate 68) delivered bicyclic molecules 69. While poor enantioselectivity was obtained with the flexible $\mathrm{C}_{2}$-symmetric Ishihara's type catalyst, the design of a much more rigid catalyst XXXIII featuring a pyridine moiety attached to a chiral benzylic center afforded the diamines with up to $86 \%$ ee. The pyridine nitrogen would coordinate iodine (III). The authors did not rule out also a possible coordination of the oxygen atom belonging to the methoxy group to iodine (III). It is worth pointing out that sodium perborate in the presence of acetic acid as an activator was the stoichiometric oxidant of choice with this catalyst instead of the commonly employed mCPBA.

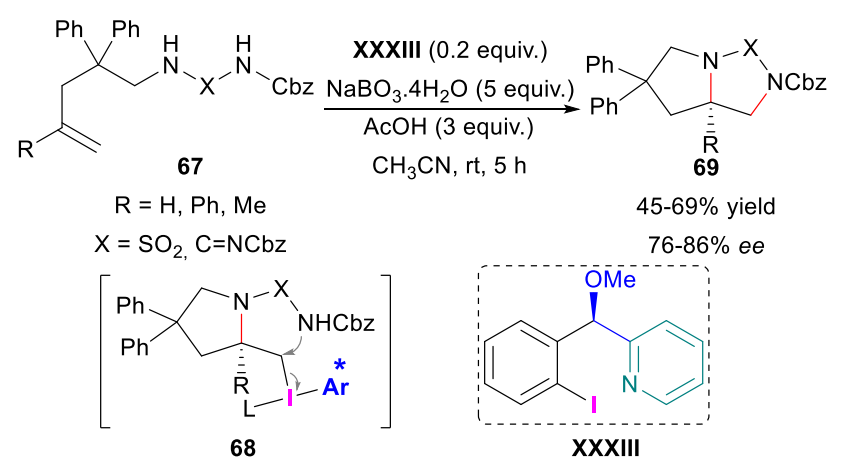

Scheme 24 Intramolecular diamination leading to dicyclized product.

\subsection{Intermolecular version}

4.2.1. Dioxygenation. In 2016, Ishihara and Muñiz et al. reported the first intermolecular asymmetric alkene dioxygenation under hypervalent iodine catalysis. ${ }^{60}$ By using $\mathrm{C}_{2}$-symmetric bislactamide XXXIV, peracetic acid as the terminal oxidant and triflic acid as a
Brønsted acid co-catalyst in acetic acid, vicinal diacetoxylation of terminal styrenes $\mathbf{7 0}$ took place in good yields with up to $94 \%$ ee (Scheme 25). X-ray analyses and ${ }^{1} \mathrm{H}$-NMR spectroscopy of the corresponding diacetate iodonium to XXXIV unveiled hydrogen bonding between the amide $\mathrm{NH}$ groups of the arms and ligand acetoxy, resulting in the creation of a supramolecular helical chirality around the central iodine (III) atom. From a mechanistic point of view, the catalytic cycle would start with the oxidation of XXXIV by peracetic acid in acetic acid to generate intermediary diacetoxyiodonium 71. Addition of triflic acid would allow the formation of a more reactive cationic catalyst state $\mathbf{7 2 ^ { 6 1 }}$ which no longer exhibits $C_{2}$-symmetry. Upon oxidation of the styrene and subsequent nucleophilic attack of acetate, intermediate $\mathbf{7 3}$ would be formed. Intramolecular nucleophilic addition of the acetate would regenerate catalyst XXXIV along with the formation of dioxolonium 74. The latter would undergo ring opening by acetate or hydrolysis to provide vicinally dioxygenated product $\mathbf{7 5}$ upon treatment with acetic anhydride.
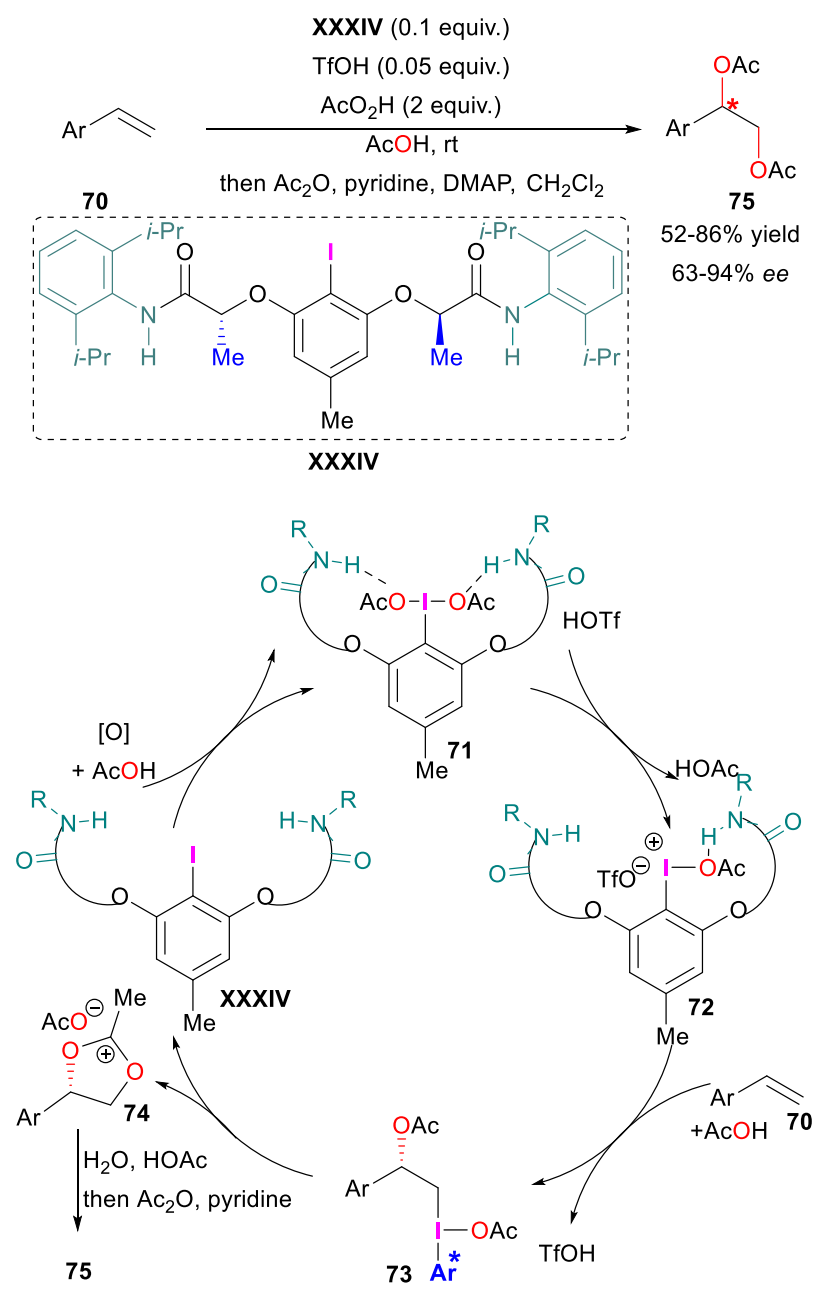

Scheme 25 Diacetoxylation of styrene.

Muñiz et al. have also demonstrated the use of bislactate $\mathbf{X X X V}$ to efficiently catalyze vicinal diacetoxylation of external and internal styrenes with up to $96 \%$ ee (Scheme 26). ${ }^{62}$ This protocol relies on the use of Selectfluor as the terminal oxidant 
(to avoid epoxidation of the alkene observed with the commonly employed peracids) and a stoichiometric amount of trimethylsilyltriflate as an iodonium co-activator.

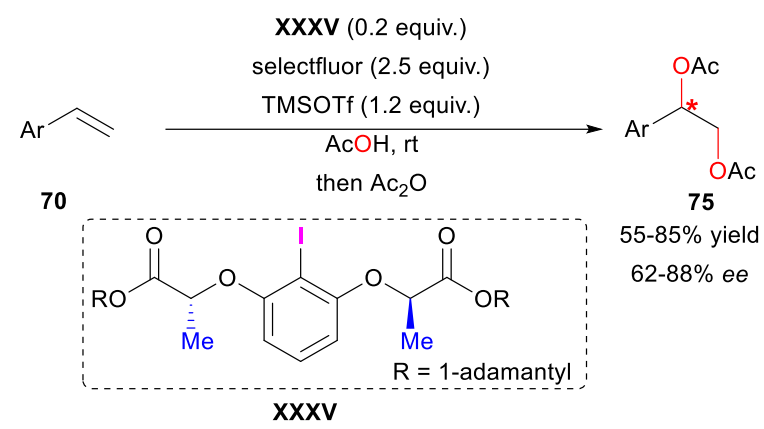

Scheme $\mathbf{2 6}$ Diacetoxylation of styrene with bis-lactate $\mathbf{X X X V}$ and selectfluor

4.2.2. Difluorination. In 2016, Jacobsen et al. described asymmetric vicinal difluorination of trisubstituted cinnamide derivative 76 in $93 \%$ ee and with excellent anti-

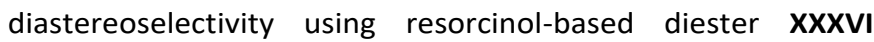
(Scheme 27, left part). ${ }^{63}$ In combination with mCPBA as stoichiometric oxidant and pyridine.9HF as nucleophilic fluorine source, the in situ generated reactive iodoarene difluoride would activate the internal alkene. Nucleophilic fluorination at the benzylic carbon would lead to anti-fluoroalkyl iodonium (III) intermediate 78. Anchimeric nucleophilic assistance by the neighboring amide group (red arrows) would allow the second fluorination to take place via intermediate $\mathbf{7 9}$ with an overall total anti-diasterosectivity to afford 1,2-difluorinated product 80. In the case of disubstituted cinnamide derivatives $77\left(R^{2}=\right.$ $\mathrm{H})$, fluoroalkyl iodonium (III) intermediate 78 would preferentially undergo aryl migration (blue arrows) via phenonium ion $\mathbf{8 1}$ to afford gem-difluoro compounds 82 in good yields and with excellent enantioselectivities using precatalyst XXXII (Scheme 27, right part). ${ }^{64}$ This elegant migratory geminal difluorination has been extended to secondary and tertiary cinnamate ester derivatives. It is worth mentioning that, in both transformations, higher enantioselectivities were obtained with aryl iodine bearing benzylic versus aliphatic substituents at the stereogenic centers probably due to attractive $\pi$-cation interactions in the transition states.

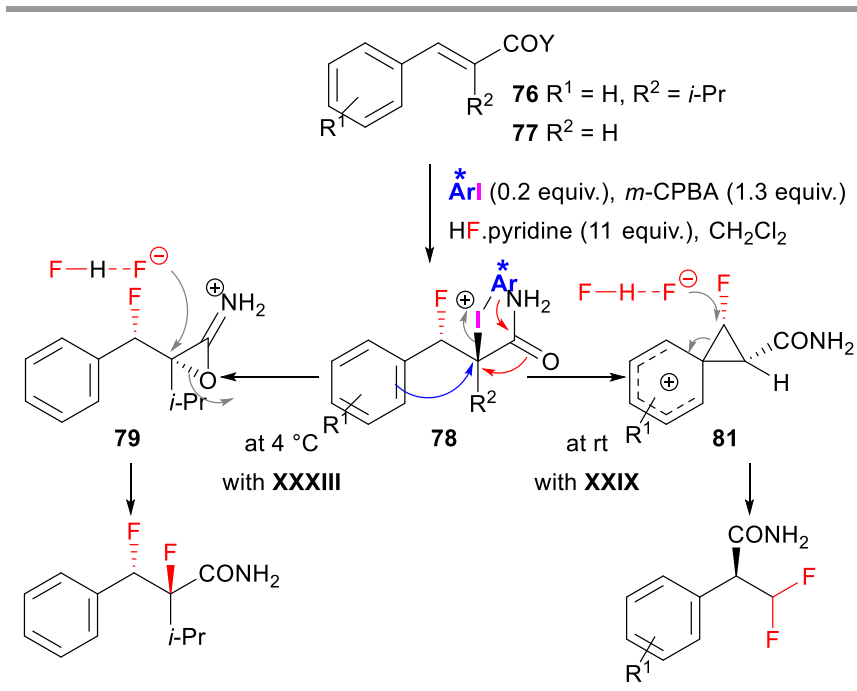

$8051 \%$ yield, $93 \%$ ee, $d r>19: 1$

$8223-83 \%$ yield, $82-96 \%$ ee

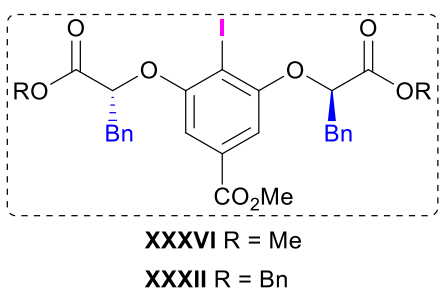

Scheme 27 Vicinal and geminal difluorination of alkenes.

Gilmour et al. reported vicinal difluorination of terminal aliphatic alkene $\mathbf{8 3}$ with modest yield and enantioselectivity using catalyst XVIII, Selectfluor as stoichiometric oxidant and amine. $5 \mathrm{HF}$ (Scheme 28). ${ }^{65}$

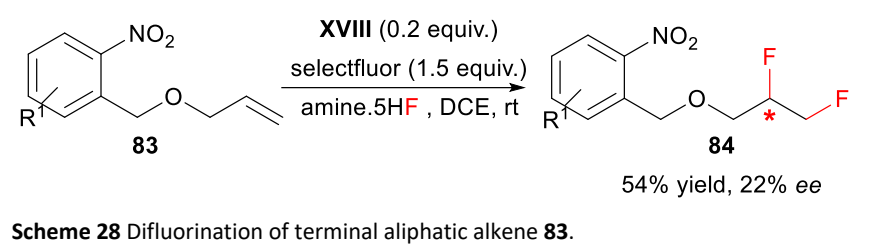

\subsubsection{Diamination}

In 2017, Muñiz et al. reported an iodine (I/III)-catalyzed enantioselective vicinal diamination of internal and external styrenes using $\mathrm{mCPBA}$ as a terminal oxidant and bismesylimide as a nitrogen source (Scheme 29). ${ }^{66}$ The undesired epoxidation background reaction was advantageously reduced when MTBE/HFIP or MTBE/TFE solvent combination were used at low temperature. Tertiary amide iodoresorcinol derivative $\mathbf{X X X V I I}$ was the catalyst of choice to provide diamines $\mathbf{8 5}$ in good yields and with excellent enantioselectivity. Since the previously observed supramolecular arrangement of resorcinol derivatives with secondary amides through intramolecular hydrogenbonding is not directly possible with this tertiary amide version, the intervention of water for the formation an analogous helical framework through intermolecular hydrogen-bonding has been proposed. From a mechanistic point of view, the authors 
suggested the initial formation of key reactive catalyst diaminoiodonium 86 which would activate the alkene. Subsequent nucleophilic addition of the bismesylimide on the benzylic carbon would give anti-vicinal aminoalkyliodonium (III) 87. Intramolecular displacement of the iodonium by the bisulfonimide with inversion of configuration would liberate the catalyst and generate cyclic intermediate $\mathbf{8 8}$. Ring opening of this intermediate would allow the second $\mathrm{C}-\mathrm{N}$ bond formation with second inversion of configuration, which would account for the anti-diastereoselectivity observed on diamines products 85.
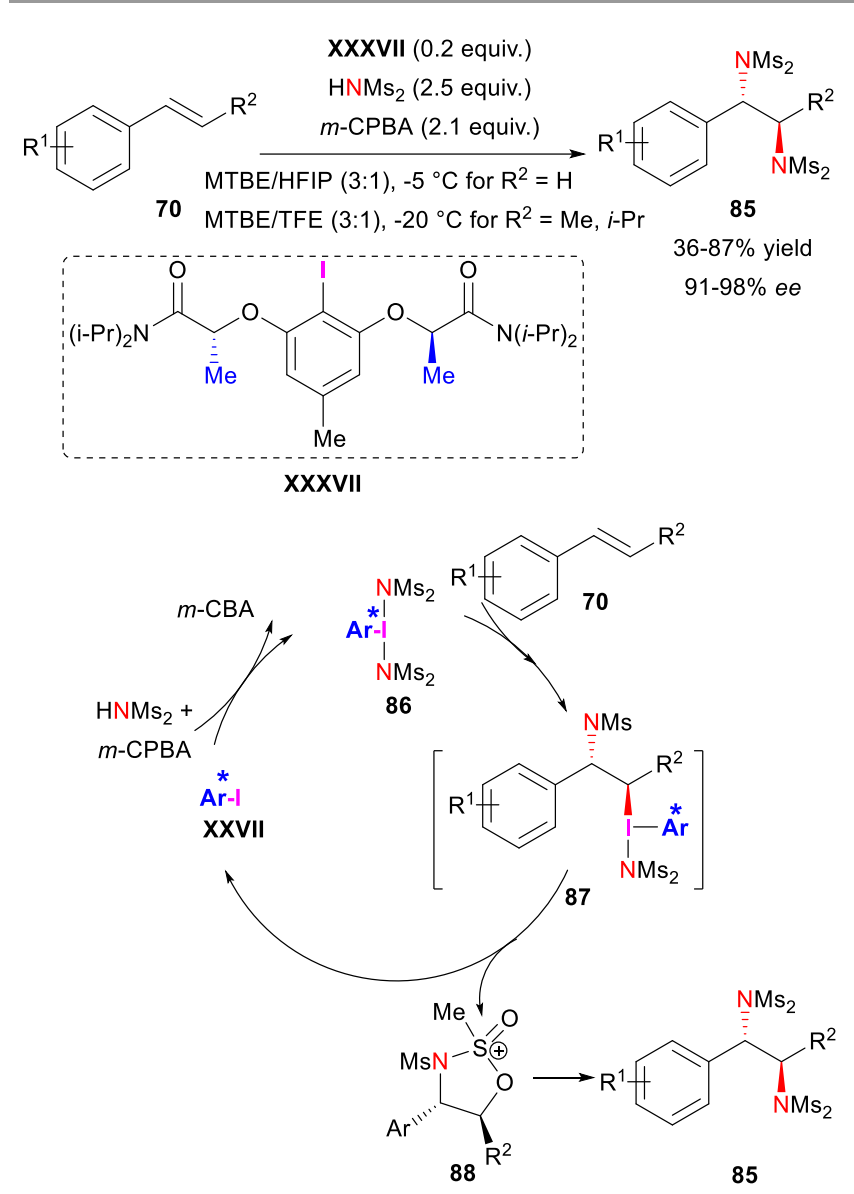

Scheme 29 Vicinal diaminations of alkenes.

\section{Conclusions}

The blossoming research field of asymmetric iodine catalysis has been summarized. Since the pioneering iodoarenes of Wirth et al. with a chiral coordinating group ortho to the iodine atom, we have seen that a handful of chiral aryliodines has been developed and used efficiently as precatalyst of hypervalent iodine (III) to perform oxidative transformations with high enantiocontrol. Although the flexible and easily tunable $\mathrm{C}_{2}$ symmetric resorcinol derivative seems to be a privileged structure in many transformations, other compounds with either central, axial or planar chirality have appeared to be also suitable precatalysts. Chiral quaternary ammonium iodides have been also ingeniously employed as precursors of hypoiodites for iodine $(-1 /+1)$ catalysis. Both such catalytic systems have proven to be ideal methods for $\alpha$ functionalization of carbonyl compounds, dearomatization of phenol derivatives and functionalization of alkenes with different type of nucleophiles (in an intra- or intermolecular way) in high enantioselectivities. Moreover, recent mechanistic investigations via X-Ray, NMR and computational analysis have allowed to shed on light on the structure of the reactive catalysts and the involved intermediaries in the catalytic cycles. These insights are expected to contribute in the near future to the design of new chiral iodine coupounds and their applications in new asymmetric reactions.

\section{Conflicts of interest}

There are no conflicts to declare.

\section{Acknowledgements}

The CNRS is gratefully acknowledged for financial supports.

\section{Notes and references}

1 a) A. Yoshimura and V. V. Zhdankin, Chem. Rev., 2016, 116, 3328-3435; b) M. S. Yusubov and V. V. Zhdankin, ResourceEfficient Technologies, 2015, 1, 49.

2 F. Berthiol, Synthesis, 2015, 47, 587

3 a) V. V. Zhdankin, Arkivoc, 2009, 1; b) S. Ghosh, S. Pradhan and I. Chatterjee, Beilstein J. Org. Chem., 2018, 14, 1244.

4 R. D. Richardson, T. K. Page, S. Altermann, S. M. Paradine, A. N. French, T. Wirth, Synlett, 2007, 538.

5 During the preparation of our manuscript, Muñiz et al. have published a review: A. Flores, E. Cots, J. Berges and K. Muñiz, Adv. Synth. Catal., 2018, 10.1002/adsc.201800521.

6 T. Hashimoto, K. Maruoka, Chem. Rev. 2007, 107, 5656.

7 M. Uyanik, K. Ishihara, ChemCatChem, 2012, 4, 177.

8 a) T. Dohi and Y. Kita, Chem. Commun. 2009, 2073; b) F. V. Singh and T. Wirth, Chem. Asian J., 2014, 9, 950.

9 Y. Yamamoto and H. Togo, Synlett, 2006, 798.

10 a) T. Wirth and U. H. Hirth, Tetrahedron: Asym., 1997, 8, 23; b) U. H. Hirt, B. Spingler and T. Wirth, J. Org. Chem., 1998, 63, 7674; c) U. H. Hirt, M. F. H. Schuster, A. N. French, O. G. Wiest and T. Wirth, Eur. J. Org. Chem., 2001, 1569.

11 S. M. Altermann, R. D. Richardson, T. K. Page, R. K. Schmidt, E. Holland, U. Mohammed, S. M. Paradine, A. N. French, C. Richter, A. M. Bahar, B. Witulski and T. Wirth, Eur. J. Org. Chem., 2008, 5315.

12 U. Farooq, S. Schäfer, A.-U.-H. A. Shah, D. M. Freudendahl and T. Wirth, Synthesis, 2010, 1023.

13 A.-A. Guilbault and C. Y. Legault, ACS Catal., 2012, 2, 219.

14 A.-A. Guilbault, B. Basdevant, V. Wanie and Cl. Y. Legault, J. Org. Chem., 2012, 77, 11283.

15 J. Yu, J. Cui, X.-S. Hou, S.-S. Liu, W.-C. Gao, S. Jiang, J. Tian and C. Zhang, Tetrahedron Asym., 2011, 22, 2039.

16 S. Brenet, F. Berthiol and J. Einhorn, Eur. J. Org. Chem., 2013, 8094.

17 S. Brenet, C. Minozzi, B. Clarens, L. Amiri and F. Berthiol, Synthesis 2015, 47, 3859.

18 (a) G. Levitre, A. Dumoulin, P. Retailleau, A. Panossian, F. R. Leroux and G. Masson, J. Org. Chem., 2017, 82, 11877. (b) J. Bortoluzzi, V. Jha, G. Levitre, M. J. Fer, J. Berreur, G. Masson, 
A. Panossian, F. R. Leroux. Org. Chem., 2018, doi: 10.1021/acs.joc.8b00648.

19 a) D. Augros, B. Yalcouye, A. Berthelot-Brehier, S. Choppin, M. Chesse, A. Panossian and F. R. Leroux, Tetrahedron, 2016, 72 5208; b) A. Berthelot-Brehier, A. Panossian, F. Colobert and F. R. Leroux, Org. Chem. Front., 2015, 2, 634; c) F. R. Leroux, A Panossian and D. C. R. Augros, Chim. 2017, 20, 682.

20 S. Beaulieu and C. Y. Legault, Chem. Eur. J. 2015, 21,11206.

21 a) B. Basdevant and C. Y. Legault, Org.Lett., 2015, 17, 4918. b) B. Basdevant, A.-A. Guilbault, S. Beaulieu, A. Jobin-Des Lauriers and C. Y. Legault, Pure Appl. Chem., 2017, 89, 781.

22 Y. Feng, R. Huang, L. Hu, Y. Xiong and V. Coeffard, Synthesis 2016, 48, 2637.

23 T. Dohi, A. Maruyama, M. Yoshimura, K. Morimoto, H. Tohma and Y. Kita, Angew. Chem. Int. Ed., 2004, 43, 3595.

24 A. Rodríguez and W. J. Moran, Synthesis, 2012, 44, 1178.

25 M. Uyanik, H. Okamoto, T. Yasui and K. Ishihara, Science, 2010, 328, 1376.

26 M. Uyanik, H. Hayashi and K. Ishihara, Science, 2014, 345, 291.

27 M. Uyanik, H. Hayashi, H. Iwata and K. Ishihara, Chem. Lett., 2016, 45, 353.

28 S. Suzuki, T. Kamo, K. Fukushi, T. Hiramatsu, E. Tokunaga, T. Dohi, Y. Kita and N. Shibata, Chem. Sci., 2014, 5, 2754.

29 R. Pluta, P. E. Krach, L. Cavallo, L. Falvienne and M. Rueping, ACS Catal., 2018, 8, 2582.

30 Y. Wang, H. Yuan, H. Lu and W.-H. Zheng, Org. Lett., 2018, 20, 2555.

31 H. Wu, Y.-P. He, L. Xu, D.-Y. Zhang and L.-Z. Gong, Angew. Chem. Int. Ed., 2014, 53, 3466.

32 A. Sreenithya, C. Patel, C. M. Hadad and R. B. Sunoj, ACS catal., 2017, 7, 4189

33 a) S. Quideau, L. Pouységu, P. A. Peixoto and D. Deffieux, Top. Curr. Chem., 2016, 373, 25. b) A. M. Harned, Tetrahedron Lett., 2014, 55, 4681.

34 a) M. Uyanik, T. Yasui, and Kazuaki Ishihara, Angew. Chem. Int. Ed., 2010, 49, 2175; b) M. Uyanik, T. Yasui and K. Ishihara, Tetrahedron, 2010, 66, 5841.

35 M. Uyanik, T. Yasui and K. Ishihara, J. Org. Chem., 2017, 82 11946.

36 M. Uyanik, T. Yasui and K. Ishihara, Angew. Chem. Int. Ed. 2013, 52, 9215.

37 S. J. Murray and H. Ibrahim, Chem. Commun., 2015, 51, 2376.

38 T. Dohi, A. Maruyama, N. Takenaga, K. Senami, Y. Minamitsuji, H. Fujioka, S. B. Caemmerer and Y. Kita, Angew. Chem. Int. Ed., 2008, 47, 3787.

39 T. Dohi, N. Takenaga, T. Nakae, Y. Toyoda, M. Yamasaki, M. Shiro, H. Fujioka, A. Maruyama and Y. Kita, J. Am. Chem. Soc., 2013, 135, 4558.

40 M. Bekkaye and G. Masson, Synthesis, 2016, 48, 302.

41 T. Dohi, H. Sasa, K. Miyazaki, M. Fujitake, N. Takenaga and Y. Kita, J. Org. Chem., 2017, 82, 11954.

42 M. Uyanik, N. Sasakura, E. Kaneko, K. Ohori and K. Ishihara, Chem. Lett., 2015, 44, 179.

43 Mechanistic studies on the activation of iodine (III) derivatives with fluorinated alcohols: I. Colomer, C. Batchelor-McAuley, B. Odell, T. J. Donohoe and R. G. Compton, J. Am. Chem. Soc. 2016, 138, 8855 .

44 M. Uyanik, N. Sasakura, M. Mizuno and K. Ishihara, ACS Catal., 2017, 7, 872 .

45 N. Jain, S. Xu, and M. A. Ciufolini, Chem. Eur. J., 2017, 23, 4542.

46 D.-Y. Zhang, L. Xu, H. Wu and L.-Z. Gong, Chem. Eur. J., 2015, 21, 10314.

47 L. Kürti, P. Herczegh, J. Visy, M. Simonyi, S. Antus and A. Pelter, J. Chem. Soc., Perkin Trans. 1, 1999, 379.

48 S. Quideau, G. Lyvinec, M. Marguerit, K. Bathany, A. Ozanne Beaudenon, T. Buffeteau, D. Cavagnat and A. Chénedé, Angew. Chem. Int. Ed., 2009, 48, 4605.

49 K. A. Volp and A. M. Harned, Chem. Commun., 2013, 49, 3001.
50 K. Muñiz and L. Fra, Synthesis, 2017, 49, 2901.

51 T. Hashimoto, Y. Shimazaki, Y. Omatsu and K. Maruoka, Angew. Chem. Int. Ed., 2018, 57, 7200.

52 R. M. Romero, T. H. Wöste and K. Muñiz, Chem. Asian J., 2014 9, 972; b) For a review on mechanistic aspects: M. Fujita, Tetrahedron Lett., 2017, 58, 4409.

53 M. Fujita, K. Mori, M. Shimogaki and T. Sugimura, Org. Lett., 2012, 14, 1294

54 M. Shimogaki, M. Fujita and T. Sugimura, Eur. J. Org. Chem., 2013, 7128 .

55 C. Gelis, A. Dumoulin, M. Bekkaye, L. Neuville and G. Masson, Org. Lett., 2017, 19, 278.

56 E. M. Woerly, S. M. Banik and E. N. Jacobsen, J. Am. Chem. Soc., 2016, 138, 13858.

57 W. Kong, P. Feige, T. de Haro and C. Nevado, Angew. Chem. Int. Ed., 2013, 52, 2469.

58 K. M. Mennie, S. M. Banik, E. C. Reichert and E. N. Jacobsen, J. Am. Chem. Soc., 2018, 140, 4797.

59 P. Mizar, A. Laverny, M. El-Sherbini, U. Farid, M. Brown, F. Malmedy and T. Wirth, Chem. Eur. J., 2014, 20, 9910.

60 S. Haubenreisser, T. H. Wöste, C. Martinez, K. Ishihara and K. Muñiz, Angew. Chem. Int. Ed., 2016, 55, 413.

61 Y.-B. Kang and L. H. Gade, J. Am. Chem. Soc., 2011, 133, 3658.

62 T. H. Wöste, K. Muñiz, Synthesis 2016, 48, 816.

63 S. M. Banik, J. W. Medley, E. N. Jacobsen, J. Am. Chem. Soc., 2016, 138, 5000.

64 S. M. Banik, J. W. Medley and E. N. Jacobsen, Science, 2016 353, 51.

65 I. G. Molnár, C. Thiehoff, M. C. Holland and R. Gilmour, ACS Catal., 2016, 6, 7167.

66 K. Muñiz, L. Barreiro, R. M. Romero and C. Martinez, J. Am. Chem. Soc., 2017, 139, 4354. 